\title{
A higher order nonlinear neutral differential equation
}

Guojing Jiang ${ }^{\mathrm{a}}$, Wei Sun ${ }^{\mathrm{b}}$, Zhefu $\mathrm{An}^{\mathrm{c}}$, Liangshi Zhao ${ }^{\mathrm{d}, *}$

${ }^{a}$ Basic Teaching Department, Vocational Technical College, Dalian, Liaoning 116035, China.

b Jiaokou No.1 Middle School, Lvliang, Shanxi 032400, China.

'School of Mathematics, Liaoning University, Shenyang, Liaoning 110036, China.

${ }^{d}$ Center for Studies of Marine Economy and Sustainable Development, Liaoning Normal University, Dalian, Liaoning 116029, China.

\begin{abstract}
This paper is concerned with the higher order nonlinear neutral differential equation

$$
\left[a(t)(x(t)+b(t) x(\tau(t)))^{\prime}\right]^{(n-1)}+f\left(t, x\left(g_{1}(t)\right), \ldots, x\left(g_{k}(t)\right)\right)=c(t), \quad t \geqslant t_{0} .
$$

By dint of the Leray-Schauder nonlinear alternative, Rothe fixed point theorem and some new techniques, we prove the existence of uncountably many bounded positive solutions for the equation. Several nontrivial examples are given to illustrate the applications and advantages of the results presented in this paper.
\end{abstract}

Keywords: Higher order nonlinear neutral differential equation, uncountably many bounded positive solutions, Leray-Schauder nonlinear alternative theorem, Rothe fixed point theorem.

2010 MSC: 34K40, 35G20.

(C)2019 All rights reserved.

\section{Introduction}

Recently, many results have been obtained on the oscillation, nonoscillation and existence of solutions for some neutral differential equations, see for example, [1-7] and the references cited therein.

Zhang et al. [4] discussed the existence of a bounded positive solution for the second order nonlinear neutral differential equation

$$
[x(t)-p(t) x(\tau(t))]^{\prime \prime}+f_{1}\left(t, x\left(\sigma_{1}(t)\right)\right)-f_{2}\left(t, x\left(\sigma_{2}(t)\right)\right)=g(t), \quad t \geqslant t_{0} .
$$

Zhou [5] obtained some new sufficient conditions for the existence of a nonoscillatory solution for the second order nonlinear neutral differential equation

$$
\left[r(t)(x(t)+p(t) x(t-\tau))^{\prime}\right]^{\prime}+\sum_{i=1}^{m} q_{i}(t) f_{i}\left(x\left(t-\sigma_{i}\right)\right)=0, \quad t \geqslant t_{0} .
$$

\footnotetext{
${ }^{*}$ Corresponding author

Email addresses: jiangguojing@qq.com (Guojing Jiang), weisun_dl@163.com (Wei Sun), zhefuan@163.com (Zhefu An), liangshizhao85@163.com (Liangshi Zhao)
}

doi: $10.22436 /$ jnsa.012.10.06

Received: 2019-02-24 Revised: 2019-04-06 Accepted: 2019-05-14 
Zhou et al. [7] proved the existence of a nonoscillatory solution for the forced higher order nonlinear neutral functional differential equation

$$
[x(t)+p(t) x(t-\tau)]^{(n)}+\sum_{i=1}^{m} q_{i}(t) f_{i}\left(x\left(t-\sigma_{i}\right)\right)=g(t), \quad t \geqslant t_{0} .
$$

Recently, Zhou and Yu [6] investigated the oscillatory behavior of the higher order nonlinear neutral forced differential equation with oscillating coefficients

$$
[x(t)-p(t) x(\tau(t))]^{(n)}+\sum_{i=1}^{m} q_{i}(t) f_{i}\left(x\left(\sigma_{i}(t)\right)\right)=s(t), \quad t \geqslant t_{0} .
$$

Liu et al. [3] studied the existence and Mann iterative approximations of nonoscillatory solutions for the nth order neutral delay differential equation

$$
[x(t)+p x(t-\tau)]^{(n)}+(-1)^{n+1} f\left(t, x\left(t-\sigma_{1}\right), \ldots, x\left(t-\sigma_{k}\right)\right)=g(t), \quad t \geqslant t_{0} .
$$

Motivated by the results in [1-7], in this paper we investigate the existence and multiplicity of bounded positive solutions for the higher order nonlinear neutral delay differential equation

$$
\left[a(t)(x(t)+b(t) x(\tau(t)))^{\prime}\right]^{(n-1)}+f\left(t, x\left(g_{1}(t)\right), \ldots, x\left(g_{k}(t)\right)\right)=c(t), \quad t \geqslant t_{0},
$$

where

(C1) $k \in \mathbb{N}, n \in \mathbb{N} \backslash\{1\}$, and $t_{0} \in \mathbb{R}$ are constants;

(C2) $a, b, c, \tau, g_{j} \in C\left(\left[t_{0},+\infty\right), \mathbb{R}\right), f \in C\left(\left[t_{0},+\infty\right) \times \mathbb{R}^{k}, \mathbb{R}\right)$ and

$$
\mathrm{a}\left(\left[\mathrm{t}_{0},+\infty\right)\right) \subseteq \mathbb{R} \backslash\{0\}, \lim _{\mathrm{t} \rightarrow+\infty} \tau(\mathrm{t})=+\infty, \lim _{\mathrm{t} \rightarrow+\infty} \mathrm{g}_{\mathrm{j}}(\mathrm{t})=+\infty, \quad j \in \mathrm{J}_{\mathrm{k}} ;
$$

(C3) $\tau$ is strictly increasing in $\left[t_{0},+\infty\right)$;

(C4) $\tau(t)<t, \quad t \in\left[t_{0},+\infty\right)$.

It is clear that Eqs. (1.1)-(1.5) are special cases of Eq. (1.6). By using the Leray-Schauder nonlinear alternative, Rothe fixed point theorem and a few new techniques, we establish several sufficient conditions for the existence of uncountably many bounded positive solutions of Eq. (1.6) under certain conditions. The results presented in this paper extend, improve and unify all results in [3-5]. Five nontrivial examples are presented to illuminate our results.

\section{Preliminaries}

Throughout this paper, we assume that $\mathbb{R}=(-\infty,+\infty), \mathbb{R}^{+}=[0,+\infty), \mathbb{N}$ stands for the sets of all positive integers,

$$
J_{k}=\{1,2, \ldots, k\}, \quad \beta_{0}=\min \left\{t_{0}, \inf _{t \geqslant t_{0}} \tau(t), \inf _{t \geqslant t_{0}} g_{j}(t): j \in J_{k}\right\} .
$$

For each $\beta \in \mathbb{R}, \mathrm{CB}([\beta,+\infty), \mathbb{R})$ denotes the Banach space of all continuous and bounded functions on $[\beta,+\infty)$ with norm $\|x\|=\sup _{t \geqslant \beta}|x(t)|$, and

$$
\begin{aligned}
\mathrm{V}(\mathrm{N}) & =\{x \in \mathrm{CB}([\beta,+\infty), \mathbb{R}): x(\mathrm{t}) \geqslant \mathrm{N}, \mathrm{t} \geqslant \beta\}, \\
\mathrm{U}(\mathrm{M}) & =\{x \in \mathrm{V}(\mathrm{N}):\|x\|<\mathrm{M}\}, \\
\mathrm{B}(\mathrm{M}, \mathrm{N}) & =\{x \in \mathrm{CB}([\beta,+\infty), \mathbb{R}):\|x-\mathrm{M}\|<\mathrm{N}\}
\end{aligned}
$$

for any $M>N>0$. It is easy to see that $V(N)$ is a closed convex subset of $C B([\beta,+\infty), \mathbb{R}), U(M)$ is a bounded open subset of $V(N)$ and $B(M, N)$ is a bounded open convex subset of $C B([\beta,+\infty), \mathbb{R})$. 
By a solution of Eq. (1.6), we mean a function $x \in C([\beta,+\infty), \mathbb{R})$ for some $\beta \geqslant\left|t_{0}\right|+\left|\beta_{0}\right|$ such that $x(t)+b(t) x(\tau(t))$ is continuously differentiable and $a(t)(x(t)+b(t) x(\tau(t)))^{\prime}$ is $n-1$ times continuously differentiable on $[\beta,+\infty)$ and such that Eq. (1.6) is satisfied for all $t \geqslant \beta$.

The following lemmas play important roles in this paper.

Lemma 2.1 (Leray-Schauder nonlinear alternative theorem, [1]). Let $\mathrm{U}$ be an open subset of a closed convex set $\mathrm{K}$ in a Banach space $\mathrm{E}$ with $\mathrm{p}^{*} \in \mathrm{U}$. Let $\mathrm{f}: \overline{\mathrm{U}} \rightarrow \mathrm{K}$ be a continuous, condensing mapping with $\mathrm{f}(\overline{\mathrm{U}})$ bounded. Then either

(a) f has a fixed point in $\overline{\mathrm{U}}$; or

(b) there exist an $\mathrm{x} \in \mathrm{\partial U}$ and $a \lambda \in(0,1)$ such that $\mathrm{x}=(1-\lambda) \mathrm{p}^{*}+\lambda \mathrm{f} x$.

Lemma $2.2([2$, Rothe fixed point theorem]). Let $\mathrm{D}$ be a bounded convex open subset of a Banach space $\mathrm{E}$ and $\mathrm{A}: \overline{\mathrm{D}} \rightarrow \mathrm{E}$ be a continuous, condensing mapping, and $\mathrm{A}(\partial \mathrm{D}) \subset \overline{\mathrm{D}}$. Then $\mathrm{A}$ has a fixed point in $\overline{\mathrm{D}}$.

Lemma 2.3. Let $\tau:\left[t_{0},+\infty\right) \rightarrow \mathbb{R}$ be continuous, $\lim _{t \rightarrow+\infty} \tau(t)=+\infty$ and (C3) and (C4) hold. Then for each $\mathrm{t} \in\left[\tau\left(\mathrm{t}_{0}\right),+\infty\right)$

$$
\left\{\tau^{-n}(t)\right\}_{n \in \mathbb{N}} \text { is strictly increasing and } \lim _{n \rightarrow \infty} \tau^{-n}(t)=+\infty \text {, }
$$

where $\tau^{-1}$ denotes the inverse function of $\tau_{,} \tau^{-n}=\tau^{-n+1}\left(\tau^{-1}\right)$ for each $n \in \mathbb{N}$ and $\tau^{0}$ is the identity function.

Proof. Since $\tau$ is continuous, it follows from (C3) that the inverse function $\tau^{-1}$ of $\tau$ exists and $\tau^{-1}$ is continuous and strictly increasing in $\left[\tau\left(t_{0}\right),+\infty\right)$. Let $t \in\left[\tau\left(t_{0}\right),+\infty\right)$. Now we show that $\lim _{n \rightarrow \infty} \tau^{-n}(t)=+\infty$. It follows from (C3) and (C4) that

$$
\tau\left(t_{0}\right) \leqslant t<\tau^{-1}(t)<\tau^{-2}(t)<\cdots<\tau^{-n}(t)<\cdots .
$$

Suppose that $\left\{\tau^{-n}(t)\right\}_{\mathfrak{n} \in \mathbb{N}}$ is bounded. In view of (2.2) we know that there exists a constant $A$ satisfying

$$
\lim _{n \rightarrow \infty} \tau^{-n}(t)=A \text {. }
$$

Using the continuity of $\tau^{-1}$ and (2.3), we get that

$$
\tau^{-1}(A)=\tau^{-1}\left(\lim _{n \rightarrow \infty} \tau^{-n}(t)\right)=\lim _{n \rightarrow \infty} \tau^{-n-1}(t)=A,
$$

which together with (C4) yields that

$$
A=\tau(A)<A,
$$

which is a contradiction. Consequently, $\left\{\tau^{-n}(t)\right\}_{\mathfrak{n} \in \mathbb{N}}$ is unbounded, which together with (2.2) yields that (2.1) holds. This completes the proof.

\section{Main results}

Now we use the Leray-Schauder nonlinear alternative theorem to show the existence and multiplicity of bounded positive solutions of Eq. (1.6).

Theorem 3.1. Let (C1) and (C2) hold. Assume that there exist four constants $\mathrm{N}, \mathrm{M}, \mathrm{b}_{*}$ and $\mathrm{b}^{*}$ and a function $\mathrm{p} \in \mathrm{C}\left(\left[\mathrm{t}_{0},+\infty\right), \mathbb{R}^{+}\right)$satisfying

$$
\begin{aligned}
& \left|f\left(t, u_{1}, u_{2}, \ldots, u_{k}\right)\right| \leqslant p(t), \quad\left(t, u_{j}\right) \in\left[t_{0},+\infty\right) \times[N, M], j \in J_{k} ; \\
& \int_{t_{0}}^{+\infty} \int_{\mu}^{+\infty} \frac{s^{n-2}}{|a(\mu)|} \max \{p(s),|c(s)|\} d s d \mu<+\infty,
\end{aligned}
$$

and

$$
0<\mathrm{N}<\left(1-\mathrm{b}_{*}-\mathrm{b}^{*}\right) \mathrm{M}, \quad \mathrm{b}_{*} \geqslant 0, \quad \mathrm{~b}^{*} \geqslant 0, \quad \mathrm{~b}_{*}+\mathrm{b}^{*}<1, \text { and }-\mathrm{b}_{*} \leqslant \mathrm{~b}(\mathrm{t}) \leqslant \mathrm{b}^{*}, \text { eventually. }
$$

Then Eq. (1.6) has uncountably many bounded positive solutions. 
Proof. Let $L \in\left(b^{*} M+N,\left(1-b_{*}\right) M\right)$. Now we prove that there exists a mapping $S_{L}: \overline{U(M)} \rightarrow V(N)$ such that it has a fixed point $x \in \overline{\mathrm{U}(M)}$, which is also a bounded positive solution of Eq. (1.6). It follows from (3.2), (3.3), and (C2) that there exist two sufficiently large numbers $T$ and $\beta$ satisfying

$$
\begin{gathered}
T>\beta \geqslant \max \left\{\left|t_{0}\right|+\left|\beta_{0}\right|, 1\right\}, \quad-b_{*} \leqslant b(t) \leqslant b^{*}, \quad t \geqslant \beta \text { and } \tau(t) \geqslant \beta, \quad t \geqslant T \\
\frac{1}{(n-2) !} \int_{\beta}^{+\infty} \int_{\mu}^{+\infty} \frac{s^{n-2}}{|a(\mu)|}[p(s)+|c(s)|] d s d \mu<\min \left\{L-b^{*} M-N,\left(1-b_{*}\right) M-L\right\} .
\end{gathered}
$$

Put $p^{*}=M-\varepsilon^{*}$, where $\varepsilon^{*} \in\left(0, \min \left\{L-b^{*} M-N,\left(1-b_{*}\right) M-L, \frac{M-N}{2}\right\}\right)$ is enough small and

$$
\frac{1}{(n-2) !} \int_{\beta}^{+\infty} \int_{\mu}^{+\infty} \frac{s^{n-2}}{|a(\mu)|}[p(s)+|c(s)|] d s d \mu<\min \left\{L-b^{*} M-N,\left(1-b_{*}\right) M-L\right\}-\varepsilon^{*} .
$$

Obviously, $\mathrm{p}^{*} \in \mathrm{U}(\mathrm{M})$. Define a mapping $\mathrm{S}_{\mathrm{L}}: \overline{\mathrm{U}(\mathrm{M})} \rightarrow \mathrm{CB}([\beta,+\infty), \mathbb{R})$ by

$$
\left(S_{L} x\right)(t)=\left(S_{1 L} x\right)(t)+\left(S_{2 L} x\right)(t), \quad \beta \leqslant t<+\infty, x \in \bar{U}(M),
$$

where the mappings $S_{1 L}, S_{2 L}: \overline{U(M)} \rightarrow C B([\beta,+\infty), \mathbb{R})$ are defined by

$$
\left(S_{1 L} x\right)(t)= \begin{cases}L-b(t) x(\tau(t)), & t \geqslant T \\ \left(S_{1 L} x\right)(T), & \beta \leqslant t<T,\end{cases}
$$

and

$$
\left(S_{2 L} x\right)(t)= \begin{cases}\frac{(-1)^{n+1}}{(n-2) !} \int_{t}^{+\infty} \int_{\mu}^{+\infty} \frac{(s-\mu)^{n-2}}{a(\mu)}\left[f\left(s, x\left(g_{1}(s)\right), \ldots, x\left(g_{k}(s)\right)\right)-c(s)\right] d s d \mu, & t \geqslant T, \\ \left(S_{2 L} x\right)(T), & \beta \leqslant t<T,\end{cases}
$$

for each $x \in \overline{\mathrm{U}(M)}$. It follows from (3.1), (3.4), and (3.6)-(3.9) that for any $x \in \overline{\mathrm{U}(M)}$ and $t \geqslant T$

$$
\begin{aligned}
S_{L} x(t)= & \left(S_{1 L} x\right)(t)+\left(S_{2 L} x\right)(t) \\
= & L-b(t) x(\tau(t)) \\
& +\frac{(-1)^{n+1}}{(n-2) !} \int_{t}^{+\infty} \int_{\mu}^{+\infty} \frac{(s-\mu)^{n-2}}{a(\mu)}\left[f\left(s, x\left(g_{1}(s)\right), \ldots, x\left(g_{k}(s)\right)\right)-c(s)\right] d s d \mu \\
\geqslant & L-b^{*} M-\frac{1}{(n-2) !} \int_{t}^{+\infty} \int_{\mu}^{+\infty} \frac{(s-\mu)^{n-2}}{|a(\mu)|}[p(s)+|c(s)|] d s d \mu \\
\geqslant & L-b^{*} M-\frac{1}{(n-2) !} \int_{T}^{+\infty} \int_{\mu}^{+\infty} \frac{(s-\mu)^{n-2}}{|a(\mu)|}[p(s)+|c(s)|] d s d \mu \\
\geqslant & L-b^{*} M-\frac{1}{(n-2) !} \int_{\beta}^{+\infty} \int_{\mu}^{+\infty} \frac{s^{n-2}}{|a(\mu)|}[p(s)+|c(s)|] d s d \mu \\
> & L-b^{*} M-\min \left\{L-b^{*} M-N,\left(1-b_{*}\right) M-L\right\}+\varepsilon^{*} \\
\geqslant & N+\varepsilon^{*}>N,
\end{aligned}
$$

which yields that $S_{\mathrm{L}}(\overline{\mathrm{U}(\mathrm{M})}) \subseteq \mathrm{V}(\mathrm{N})$.

Next we show that $S_{2 L}: \overline{\mathrm{U}(M)} \rightarrow \mathrm{CB}([\beta,+\infty), \mathbb{R})$ is a continuous and relatively compact mapping.

Let $\left\{x_{m}\right\}_{m \in \mathbb{N}} \subseteq \overline{\mathrm{U}(M)}$ and $x \in \overline{\mathrm{U}(M)}$ with $\lim _{m \rightarrow \infty} x_{m}=x$. By virtue of (3.1), (3.6), (3.9), and the continuity of $f$, we infer that

$$
\left\|S_{2 L} x_{m}-S_{2 L} x\right\|=\sup _{t \geqslant \beta} \mid \frac{(-1)^{n+1}}{(n-2) !} \int_{t}^{+\infty} \int_{\mu}^{+\infty} \frac{(s-\mu)^{n-2}}{a(\mu)}\left[f\left(s, x_{m}\left(g_{1}(s)\right), \ldots, x_{m}\left(g_{k}(s)\right)\right)\right.
$$




$$
\begin{aligned}
&\left.-f\left(s, x\left(g_{1}(s)\right), \ldots, x\left(g_{k}(s)\right)\right)\right] d s d \mu \mid \\
& \leqslant \frac{1}{(n-2) !} \int_{T}^{+\infty} \int_{\mu}^{+\infty} \frac{s^{n-2}}{|a(\mu)|}\left|f\left(s, x_{m}\left(g_{1}(s)\right), \ldots, x_{m}\left(g_{k}(s)\right)\right)-f\left(s, x\left(g_{1}(s)\right), \ldots, x\left(g_{k}(s)\right)\right)\right| d s d \mu \\
& \leqslant \frac{1}{(n-2) !} \int_{\beta}^{+\infty} \int_{\mu}^{+\infty} \frac{s^{n-2}}{|a(\mu)|} \mid f\left(s, x_{m}\left(g_{1}(s)\right), \ldots, x_{m}\left(g_{k}(s)\right)\right) \\
&-f\left(s, x\left(g_{1}(s)\right), \ldots, x\left(g_{k}(s)\right)\right) \mid d s d \mu, \quad m \in \mathbb{N}, \\
& \int_{\mu}^{+\infty} \frac{s^{n-2}}{|a(\mu)|}\left|f\left(s, x_{m}\left(g_{1}(s)\right), \ldots, x_{m}\left(g_{k}(s)\right)\right)-f\left(s, x\left(g_{1}(s)\right), \ldots, x\left(g_{k}(s)\right)\right)\right| d s \\
& \leqslant 2 \int_{\mu}^{+\infty} \frac{s^{n-2}}{|a(\mu)|} p(s) d s, \quad \mu \in[\beta,+\infty)
\end{aligned}
$$

and

$$
\left|f\left(s, x_{m}\left(g_{1}(s)\right), \ldots, x_{m}\left(g_{k}(s)\right)\right)-f\left(s, x\left(g_{1}(s)\right), \ldots, x\left(g_{k}(s)\right)\right)\right| \rightarrow 0 \quad \text { as } m \rightarrow \infty, \quad s \in[\beta,+\infty),
$$

which together with the Lebesgue dominated convergence theorem yield that

$$
\lim _{m \rightarrow \infty}\left\|S_{2 L} x_{m}-S_{2 L} x\right\|=0,
$$

that is, $S_{2 L}$ is continuous in $\overline{\mathrm{U}(M)}$.

In light of (3.1), (3.6), and (3.9), we deduce that for all $x \in \overline{\mathrm{U}(\mathrm{M})}$

$$
\begin{aligned}
\left\|S_{2 L} x\right\|=\sup _{t \geqslant \beta}\left|S_{2 L} x(t)\right| & =\sup _{t \geqslant T}\left|\frac{(-1)^{n+1}}{(n-2) !} \int_{t}^{+\infty} \int_{\mu}^{+\infty} \frac{(s-\mu)^{n-2}}{a(\mu)}\left[f\left(s, x\left(g_{1}(s)\right), \ldots, x\left(g_{k}(s)\right)\right)-c(s)\right] d s d \mu\right| \\
& \leqslant \frac{1}{(n-2) !} \int_{T}^{+\infty} \int_{\mu}^{+\infty} \frac{s^{n-2}}{|a(\mu)|}[p(s)+|c(s)|] d s d \mu \\
& \leqslant \frac{1}{(n-2) !} \int_{\beta}^{+\infty} \int_{\mu}^{+\infty} \frac{s^{n-2}}{|a(\mu)|}[p(s)+|c(s)|] d s d \mu \\
& <\min \left\{L-b^{*} M-N,\left(1-b_{*}\right) M-L\right\}-\varepsilon^{*}<M,
\end{aligned}
$$

which means that $S_{2 L}(\overline{\mathrm{U}(M)})$ is uniformly bounded in $[\beta,+\infty)$.

Let $\varepsilon>0$. Notice that (3.2) ensures that there exists $T^{*}>T$ satisfying

$$
\frac{1}{(n-2) !} \int_{T^{*}}^{+\infty} \int_{\mu}^{+\infty} \frac{s^{n-2}}{|a(\mu)|}[p(s)+|c(s)|] d s d \mu<\frac{\varepsilon}{2}
$$

which together with (3.1) and (3.9) yields that for all $x \in \overline{\mathrm{U}(M)}$ and $t_{2}>t_{1} \geqslant T^{*}$

$$
\begin{aligned}
\left|\left(S_{2 L} x\right)\left(t_{2}\right)-\left(S_{2 L} x\right)\left(t_{1}\right)\right|= & \mid \frac{(-1)^{n+1}}{(n-2) !} \int_{t_{2}}^{+\infty} \int_{\mu}^{+\infty} \frac{(s-\mu)^{n-2}}{a(\mu)}\left[f\left(s, x\left(g_{1}(s)\right), \ldots, x\left(g_{k}(s)\right)\right)-c(s)\right] d s d \mu \\
& -\frac{(-1)^{n+1}}{(n-2) !} \int_{t_{1}}^{+\infty} \int_{\mu}^{+\infty} \frac{(s-\mu)^{n-2}}{a(\mu)}\left[f\left(s, x\left(g_{1}(s)\right), \ldots, x\left(g_{k}(s)\right)\right)-c(s)\right] d s d \mu \mid \\
\leqslant & \frac{1}{(n-2) !}\left[\int_{t_{2}}^{+\infty} \int_{\mu}^{+\infty} \frac{s^{n-2}}{|a(\mu)|}[p(s)+|c(s)|] d s d \mu\right. \\
& \left.+\int_{t_{1}}^{+\infty} \int_{\mu}^{+\infty} \frac{s^{n-2}}{|a(\mu)|}[p(s)+|c(s)|] d s d \mu\right] \\
\leqslant & \frac{2}{(n-2) !} \int_{T^{*}}^{+\infty} \int_{\mu}^{+\infty} \frac{s^{n-2}}{|a(\mu)|}[p(s)+|c(s)|] d s d \mu<\varepsilon .
\end{aligned}
$$


Put

$$
M_{1}=\max \left\{\frac{1}{(n-2) !} \int_{\mu}^{+\infty} \frac{s^{n-2}}{|a(\mu)|}[p(s)+|c(s)|] d s: T \leqslant \mu \leqslant T^{*}\right\} \text { and } \delta=\frac{\varepsilon}{1+M_{1}} .
$$

It follows from (3.1) and (3.9) that for all $x \in \overline{\mathrm{U}(\mathrm{M})}$ and $\mathrm{t}_{1}, \mathrm{t}_{2} \in\left[\mathrm{T}, \mathrm{T}^{*}\right]$ with $\mathrm{t}_{1} \leqslant \mathrm{t}_{2}<\mathrm{t}_{1}+\delta$

$$
\begin{aligned}
&\left|\left(S_{2 L} x\right)\left(t_{2}\right)-\left(S_{2 L} x\right)\left(t_{1}\right)\right| \\
&=\mid \frac{(-1)^{n+1}}{(n-2) !} \int_{t_{2}}^{+\infty} \int_{\mu}^{+\infty} \frac{(s-\mu)^{n-2}}{a(\mu)}\left[f\left(s, x\left(g_{1}(s)\right), \ldots, x\left(g_{k}(s)\right)\right)-c(s)\right] d s d \mu \\
&-\frac{(-1)^{n+1}}{(n-2) !} \int_{t_{1}}^{+\infty} \int_{\mu}^{+\infty} \frac{(s-\mu)^{n-2}}{a(\mu)}\left[f\left(s, x\left(g_{1}(s)\right), \ldots, x\left(g_{k}(s)\right)\right)-c(s)\right] d s d \mu \mid \\
& \leqslant \frac{1}{(n-2) !} \int_{t_{1}}^{t_{2}} \int_{\mu}^{+\infty} \frac{(s-\mu)^{n-2}}{|a(\mu)|}\left|f\left(s, x\left(g_{1}(s)\right), \ldots, x\left(g_{k}(s)\right)\right)-c(s)\right| d s d \mu \\
& \leqslant \frac{1}{(n-2) !} \int_{t_{1}}^{t_{2}} \int_{\mu}^{+\infty} \frac{s^{n-2}}{|a(\mu)|}[p(s)+|c(s)|] d s d \mu \\
& \leqslant M_{1}\left|t_{2}-t_{1}\right|<\varepsilon .
\end{aligned}
$$

By virtue of (3.9), we get that for all $x \in \overline{\mathrm{U}(M)}$ and $t_{1}, t_{2} \in[\beta, T]$

$$
\left|\left(S_{2 L} x\right)\left(t_{2}\right)-\left(S_{2 L} x\right)\left(t_{1}\right)\right|=\left|\left(S_{2 L} x\right)(T)-\left(S_{2 L} x\right)(T)\right|=0<\varepsilon .
$$

It follows from (3.10)-(3.12) that $S_{2 L}(\overline{\mathrm{U}(M)})$ is equicontinuous in $[\beta,+\infty)$. Thus $S_{2 L}(\overline{\mathrm{U}(M)})$ is relatively compact. In view of (3.4) and (3.8), we infer that for all $x, y \in \overline{\mathrm{U}(M)}$ and $t \geqslant T$

$$
\left|\left(S_{1 \mathrm{~L}} x\right)(t)-\left(S_{1 L} y\right)(t)\right|=\left|b(t)\left\|x(\tau(t))-y(\tau(t)) \mid \leqslant\left(b_{*}+b^{*}\right)\right\| x-y \|,\right.
$$

which yields that

$$
\left\|S_{1 \mathrm{~L}} x-S_{1 \mathrm{~L}} y\right\| \leqslant\left(b_{*}+b^{*}\right)\|x-y\|
$$

that is, $S_{1 L}$ is a contraction mapping in $\overline{\mathrm{U}(M)}$ and $\left\|S_{1 L}(\overline{\mathrm{U}(M)})\right\| \leqslant 2 M\left(b_{*}+b^{*}\right)$. Hence $S_{L}$ is a continuous, condensing mapping and $S_{\mathrm{L}}(\overline{\mathrm{U}(\mathrm{M})})$ is bounded. Put

$$
P=\{x \in C B([\beta,+\infty), \mathbb{R}): N \leqslant x(t) \leqslant M, t \geqslant \beta \text { and }\|x\|=M\}
$$

and

$$
Q=\left\{x \in C B([\beta,+\infty), \mathbb{R}): N \leqslant x(t) \leqslant M, \quad t \geqslant \beta \text { and there exists } t^{*} \geqslant \beta \text { satisfying } x\left(t^{*}\right)=N\right\} .
$$

It is easy to see that $\partial \mathrm{U}(M)=\mathrm{P} \cup \mathrm{Q}$. Suppose that there exist $x \in \partial \mathrm{U}(\mathrm{M})$ and $\lambda \in(0,1)$ with

$$
x=(1-\lambda) p^{*}+\lambda S_{L} x .
$$

Now we consider two possible cases as follows:

Case 1. Let $x \in$ P. It follows from (3.4), (3.6)-(3.9), (3.13), and (3.15) that

$$
\begin{aligned}
x(t)= & (1-\lambda) p^{*}+\lambda S_{L} x(t) \\
= & (1-\lambda) p^{*}+\lambda\left[L-b(t) x(\tau(t))+\frac{(-1)^{n+1}}{(n-2) !} \int_{t}^{+\infty} \int_{\mu}^{+\infty} \frac{(s-\mu)^{n-2}}{a(\mu)}\right. \\
& \left.\times\left[f\left(s, x\left(g_{1}(s)\right), \ldots, x\left(g_{k}(s)\right)\right)-c(s)\right] d s d \mu\right] \\
\leqslant & (1-\lambda)\left(M-\varepsilon^{*}\right)+\lambda\left[L+b_{*} M+\frac{1}{(n-2) !} \int_{T}^{+\infty} \int_{\mu}^{+\infty} \frac{s^{n-2}}{|a(\mu)|}[p(s)+|c(s)|] d s d \mu\right]
\end{aligned}
$$




$$
\begin{aligned}
& <(1-\lambda)\left(M-\varepsilon^{*}\right)+\lambda\left[L+b_{*} M+\min \left\{L-b^{*} M-N,\left(1-b_{*}\right) M-L\right\}-\varepsilon^{*}\right] \\
& \leqslant M-\varepsilon^{*}, \quad t \geqslant T
\end{aligned}
$$

which implies that

$$
M=\|x\|=\sup _{t \geqslant \beta}|x(t)| \leqslant M-\varepsilon^{*}<M
$$

which is a contradiction;

Case 2. Let $x \in \mathrm{Q}$. It follows from (3.4), (3.6)-(3.9), (3.14), and (3.15) that

$$
\begin{aligned}
N=x\left(t^{*}\right)= & (1-\lambda) p^{*}+\lambda S_{L} x\left(t^{*}\right) \\
= & (1-\lambda) p^{*}+\lambda\left[L-b\left(t^{*}\right) x\left(\tau\left(t^{*}\right)\right)+\frac{(-1)^{n+1}}{(n-2) !} \int_{\max \left\{t^{*}, T\right\}}^{+\infty} \int_{\mu}^{+\infty} \frac{(s-\mu)^{n-2}}{a(\mu)}\right. \\
& \left.\times\left[f\left(s, x\left(g_{1}(s)\right), \ldots, x\left(g_{k}(s)\right)\right)-c(s)\right] d s d \mu\right] \\
\geqslant & (1-\lambda)\left(M-\varepsilon^{*}\right)+\lambda\left[L-b^{*} M-\frac{1}{(n-2) !} \int_{\max \left\{t^{*}, T\right\}}^{+\infty} \int_{\mu}^{+\infty} \frac{s^{n-2}}{|a(\mu)|}[p(s)+|c(s)|] d s d \mu\right] \\
> & (1-\lambda)\left(M-\varepsilon^{*}\right)+\lambda\left[L-b^{*} M-\min \left\{L-b^{*} M-N,\left(1-b_{*}\right) M-L\right\}+\varepsilon^{*}\right] \\
\geqslant & (1-\lambda)\left(M-\varepsilon^{*}\right)+\lambda\left(N+\varepsilon^{*}\right) \\
\geqslant & \min \left\{M-\varepsilon^{*}, N+\varepsilon^{*}\right\}=N+\varepsilon^{*},
\end{aligned}
$$

which is absurd. Thus Lemma 2.1 ensures that there exists $x \in \overline{U(M)}$ satisfying $S_{L} x=S_{1 L} x+S_{2 L} x=x$, that is,

$$
x(t)=L-b(t) x(\tau(t))+\frac{(-1)^{n+1}}{(n-2) !} \int_{t}^{+\infty} \int_{\mu}^{+\infty} \frac{(s-\mu)^{n-2}}{a(\mu)}\left[f\left(s, x\left(g_{1}(s)\right), \ldots, x\left(g_{k}(s)\right)\right)-c(s)\right] d s d \mu, \quad t \geqslant T,
$$

which means that

$$
a(t)(x(t)+b(t) x(\tau(t)))^{\prime}=\frac{(-1)^{n}}{(n-2) !} \int_{t}^{+\infty}(s-t)^{n-2}\left[f\left(s, x\left(g_{1}(s)\right), \ldots, x\left(g_{k}(s)\right)\right)-c(s)\right] d s, \quad t \geqslant T,
$$

which yields that

$$
{ }^{(n-2)}=(-1)^{2} \int_{t}^{+\infty}\left[f\left(s, x\left(g_{1}(s)\right), \ldots, x\left(g_{k}(s)\right)\right)-c(s)\right] d s, \quad t \geqslant T
$$

and

$$
\left[a(t)(x(t)+b(t) x(\tau(t)))^{\prime}\right]^{(n-1)}=-f\left(t, x\left(g_{1}(t)\right), \ldots, x\left(g_{k}(t)\right)\right)+c(t), \quad t \geqslant T,
$$

that is, $x$ is a bounded positive solution of Eq. (1.6) in $\overline{\mathrm{U}(M)}$.

Finally we show that Eq. (1.6) has uncountably many bounded positive solutions in $\overline{\mathrm{U}(\mathrm{M})}$. Let $\mathrm{L}_{1}, \mathrm{~L}_{2} \in\left(\mathrm{b}^{*} \mathrm{M}+\mathrm{N},\left(1-\mathrm{b}_{*}\right) \mathrm{M}\right)$ and $\mathrm{L}_{1} \neq \mathrm{L}_{2}$. Similarly we infer that for each $\theta \in\{1,2\}$, there exists a mapping $S_{L_{\theta}}: \overline{U(M)} \rightarrow V(N)$ satisfying (3.4)-(3.9), where $L, \beta, T, S_{1 L}, S_{2 L}$, and $S_{L}$ are replaced by $L_{\theta}, \beta_{\theta}, T_{L_{\theta}}, S_{1 L_{\theta}}, S_{2 L_{\theta}}$, and $S_{L_{\theta}}$, respectively, and the mapping $S_{L_{\theta}}$ has a fixed point $x^{\theta} \in \bar{U}(M)$, which is a bounded positive solution of Eq. (1.6) in $\overline{U(M)}$, that is,

$$
\begin{aligned}
x^{\theta}(t)= & L_{\theta}-b(t) x^{\theta}(\tau(t))+\frac{(-1)^{n+1}}{(n-2) !} \int_{t}^{+\infty} \int_{\mu}^{+\infty} \frac{(s-\mu)^{n-2}}{a(\mu)} \\
& \times\left[f\left(s, x^{\theta}\left(g_{1}(s)\right), \ldots, x^{\theta}\left(g_{k}(s)\right)\right)-c(s)\right] d s d \mu, \quad t \geqslant T_{L_{\theta}} .
\end{aligned}
$$


It follows from (3.2) that there exists $T_{*}>\max \left\{T_{L_{1}}, T_{L_{2}}\right\}$ satisfying

$$
\frac{1}{(\mathrm{n}-2) !} \int_{\mathrm{T}_{*}}^{+\infty} \int_{\mu}^{+\infty} \frac{\mathrm{s}^{\mathrm{n}-2}}{|\mathrm{a}(\mu)|} \mathrm{p}(\mathrm{s}) \mathrm{d} \mathrm{d} \mathrm{d} \mu<\frac{\left|\mathrm{L}_{1}-\mathrm{L}_{2}\right|}{4} .
$$

In order to prove that the set of bounded positive solutions of Eq. (1.6) is uncountable, it is sufficient to verify that $x^{1} \neq x^{2}$. In terms of (3.1), (3.16), and (3.17), we deduce that for $t \geqslant T_{*}$

$$
\begin{aligned}
\left|x^{1}(t)-x^{2}(t)\right|= & \mid L_{1}-L_{2}-b(t) x^{1}(\tau(t))+b(t) x^{2}(\tau(t))+\frac{(-1)^{n+1}}{(n-2) !} \int_{t}^{+\infty} \int_{\mu}^{+\infty} \frac{(s-\mu)^{n-2}}{a(\mu)} \\
& \quad \times\left[f\left(s, x^{1}\left(g_{1}(s)\right), \ldots, x^{1}\left(g_{k}(s)\right)\right)-f\left(s, x^{2}\left(g_{1}(s)\right), \ldots, x^{2}\left(g_{k}(s)\right)\right)\right] d s d \mu \mid \\
\geqslant & \left|L_{1}-L_{2}\right|-|b(t)|\left|x^{1}(\tau(t))-x^{2}(\tau(t))\right|-\frac{1}{(n-2) !} \int_{t}^{+\infty} \int_{\mu}^{+\infty} \frac{(s-\mu)^{n-2}}{|a(\mu)|} \\
& \times\left[\mid f\left(s, x^{1}\left(g_{1}(s)\right), \ldots, x^{1}\left(g_{k}(s)\right)|+| f\left(s, x^{2}\left(g_{1}(s)\right), \ldots, x^{2}\left(g_{k}(s)\right)\right) \mid\right] d s d \mu\right. \\
\geqslant & \left|L_{1}-L_{2}\right|-\left(b_{*}+b^{*}\right)\left\|x^{1}-x^{2}\right\|-\frac{2}{(n-2) !} \int_{T_{*}}^{+\infty} \int_{\mu}^{+\infty} \frac{s^{n-2}}{|a(\mu)|} p(s) d s d \mu \\
> & \frac{1}{2}\left|L_{1}-L_{2}\right|-\left(b_{*}+b^{*}\right)\left\|x^{1}-x^{2}\right\|,
\end{aligned}
$$

which means that

$$
\left\|x^{1}-x^{2}\right\|>\frac{\left|L_{1}-L_{2}\right|}{2\left(1+b_{*}+b^{*}\right)}>0,
$$

that is, $x^{1} \neq x^{2}$. This completes the proof.

Theorem 3.2. Let (C1), (C2), and (C3) hold. Assume that there exist four constants $\mathrm{N}, \mathrm{M}, \mathrm{b}_{*}$ and $\mathrm{b}^{*}$ and $a$ function $\mathrm{p} \in \mathrm{C}\left(\left[\mathrm{t}_{0},+\infty\right), \mathbb{R}^{+}\right)$satisfying (3.1), (3.2), and

$$
0<\mathrm{Nb}^{*} \mathrm{~b}_{*}<\mathrm{M}\left(\mathrm{b}_{*}^{2}-\mathrm{b}^{*}\right), \quad 1<\mathrm{b}_{*} \leqslant \mathrm{~b}(\mathrm{t}) \leqslant \mathrm{b}^{*}<\mathrm{b}_{*}^{2} \text {, eventually. }
$$

Then Eq. (1.6) has uncountably many bounded positive solutions.

Proof. Let $\mathrm{L} \in\left(\frac{\mathrm{b}^{*}}{\mathrm{~b}_{*}} M+\mathrm{b}^{*} \mathrm{~N}, \mathrm{~b}_{*} M\right)$. First of all we prove that there exists a mapping $S_{\mathrm{L}}: \overline{\mathrm{U}(M)} \rightarrow \mathrm{V}(\mathrm{N})$ such that it has a fixed point $x \in \overline{\mathrm{U}(M)}$, which is also a bounded positive solution of Eq. (1.6). Note that (C2) and (C3) imply that the inverse function $\tau^{-1}$ of $\tau$ is strictly increasing and continuous in $\left[\tau\left(t_{0}\right),+\infty\right)$ and $\lim _{t \rightarrow+\infty} \tau^{-1}(t)=+\infty$. It follows from (3.2) and (3.18) that there exist constants $T$ and $\beta$ satisfying

$$
\begin{aligned}
& \min \left\{\tau^{-1}(T), T\right\}>\beta \geqslant \max \left\{1,\left|t_{0}\right|+\left|\beta_{0}\right|,\left|\tau\left(t_{0}\right)\right|\right\}, \quad 1<b_{*} \leqslant b(t) \leqslant b^{*}<b_{* \prime}^{2}, \quad t \geqslant \beta ; \\
& \frac{1}{(n-2) !} \int_{\beta}^{+\infty} \int_{\mu}^{+\infty} \frac{s^{n-2}}{|a(\mu)|}[p(s)+|c(s)|] d s d \mu<\min \left\{b_{*} M-L, \frac{b_{*} L}{b^{*}}-M-b_{*} N\right\} .
\end{aligned}
$$

Let $p^{*}=M-\varepsilon^{*}$, where $\varepsilon^{*} \in\left(0, \min \left\{b_{*} M-L, \frac{b_{*} L}{b^{*}}-M-b_{*} N, \frac{b_{*}(M-N)}{1+b_{*}}\right\}\right)$ is enough small and

$$
\frac{1}{(n-2) !} \int_{\beta}^{+\infty} \int_{\mu}^{+\infty} \frac{s^{n-2}}{|a(\mu)|}[p(s)+|c(s)|] d s d \mu<\min \left\{b_{*} M-L, \frac{b_{*} L}{b^{*}}-M-b_{*} N\right\}-\varepsilon^{*}
$$

Clearly $p^{*} \in \mathrm{U}(M)$. Define a mapping $S_{L}: \bar{U}(M) \rightarrow C B([\beta,+\infty), \mathbb{R})$ by (3.7), where the mappings $\mathrm{S}_{1 \mathrm{~L}}, \mathrm{~S}_{2 \mathrm{~L}}: \overline{\mathrm{U}(\mathrm{M})} \rightarrow \mathrm{CB}([\beta,+\infty), \mathbb{R})$ are defined by

$$
\left(S_{1 L} x\right)(t)= \begin{cases}\frac{L}{b\left(\tau^{-1}(t)\right)}-\frac{x\left(\tau^{-1}(t)\right)}{b\left(\tau^{-1}(t)\right)}, & t \geqslant T, \\ \left(S_{1 L} x\right)(T), & \beta \leqslant t<T,\end{cases}
$$


and

$$
\left(S_{2 L} x\right)(t)=\left\{\begin{array}{l}
\frac{(-1)^{n+1}}{b\left(\tau^{-1}(t)\right)(n-2) !} \int_{\tau^{-1}(t)}^{+\infty} \int_{\mu}^{+\infty} \frac{(s-\mu)^{n-2}}{a(\mu)}\left[f\left(s, x\left(g_{1}(s)\right), \ldots, x\left(g_{k}(s)\right)\right)-c(s)\right] d s d \mu, t \geqslant T, \\
\left(S_{2 L} x\right)(T), \\
\beta \leqslant t<T,
\end{array}\right.
$$

for each $x \in \overline{\mathrm{U}(M)}$. In view of (3.1), (3.7), (3.19), and (3.21)-(3.23), we infer that for any $x \in \overline{\mathrm{U}(M)}$ and $t \geqslant T$

$$
\begin{aligned}
S_{\mathrm{L}} x(t)= & \left(S_{1 \mathrm{~L}} x\right)(\mathrm{t})+\left(S_{2 \mathrm{~L}} x\right)(\mathrm{t}) \\
= & \frac{\mathrm{L}}{\mathrm{b}\left(\tau^{-1}(\mathrm{t})\right)}-\frac{x\left(\tau^{-1}(\mathrm{t})\right)}{\mathrm{b}\left(\tau^{-1}(\mathrm{t})\right)}+\frac{(-1)^{n+1}}{b\left(\tau^{-1}(\mathrm{t})\right)(\mathrm{n}-2) !} \int_{\tau^{-1}(\mathrm{t})}^{+\infty} \int_{\mu}^{+\infty} \frac{(s-\mu)^{n-2}}{a(\mu)} \\
& \times\left[f\left(s, x\left(g_{1}(s)\right), \ldots, x\left(g_{k}(s)\right)\right)-c(s)\right] d s d \mu \\
\geqslant & \frac{L}{b^{*}}-\frac{M}{b_{*}}-\frac{1}{b_{*}(n-2) !} \int_{\tau^{-1}(T)}^{+\infty} \int_{\mu}^{+\infty} \frac{(s-\mu)^{n-2}}{|a(\mu)|}[p(s)+|c(s)|] d s d \mu \\
\geqslant & \frac{L}{b^{*}}-\frac{M}{b_{*}}-\frac{1}{b_{*}(n-2) !} \int_{\beta}^{+\infty} \int_{\mu}^{+\infty} \frac{s^{n-2}}{|a(\mu)|}[p(s)+|c(s)|] d s d \mu \\
\geqslant & \frac{L}{b^{*}}-\frac{M}{b_{*}}-\frac{1}{b_{*}} \min \left\{b_{*} M-L, \frac{b_{*} L}{b^{*}}-M-b_{*} N\right\}+\frac{\varepsilon^{*}}{b_{*}} \\
\geqslant & N+\frac{\varepsilon^{*}}{b_{*}}>N,
\end{aligned}
$$

which implies that $\mathrm{S}_{\mathrm{L}}(\overline{\mathrm{U}(\mathrm{M})}) \subseteq \mathrm{V}(\mathrm{N})$.

Secondly we prove that $S_{2 L}: \overline{U(M)} \rightarrow \mathrm{CB}([\beta,+\infty), \mathbb{R})$ is a continuous and relatively compact mapping.

Let $\left\{x_{m}\right\}_{m \in \mathbb{N}} \subseteq \overline{\mathrm{U}(M)}$ and $x \in \overline{\mathrm{U}(M)}$ with $\lim _{m \rightarrow \infty} x_{m}=x$. It follows from (3.1), (3.19), (3.21), (3.23), and the continuity of $f$ that

$$
\begin{aligned}
&\left\|S_{2 L} x_{m}-S_{2 L} x\right\|= \sup _{t \geqslant \beta} \mid \frac{(-1)^{n+1}}{b\left(\tau^{-1}(t)\right)(n-2) !} \int_{\tau^{-1}(t)}^{+\infty} \int_{\mu}^{+\infty} \frac{(s-\mu)^{n-2}}{a(\mu)}\left[f\left(s, x_{m}\left(g_{1}(s)\right), \ldots, x_{m}\left(g_{k}(s)\right)\right)\right. \\
&\left.-f\left(s, x\left(g_{1}(s)\right), \ldots, x\left(g_{k}(s)\right)\right)\right] d s d \mu \mid \\
& \leqslant \frac{1}{b_{*}(n-2) !} \int_{\tau^{-1}(T)}^{+\infty} \int_{\mu}^{+\infty} \frac{s^{n-2}}{|a(\mu)|} \mid f\left(s, x_{n}\left(g_{1}(s)\right), \ldots, x_{n}\left(g_{k}(s)\right)\right) \\
&-f\left(s, x\left(g_{1}(s)\right), \ldots, x\left(g_{k}(s)\right)\right) \mid d s d \mu \\
& \leqslant \frac{1}{b_{*}(n-2) !} \int_{\beta}^{+\infty} \int_{\mu}^{+\infty} \frac{s^{n-2}}{|a(\mu)|} \mid f\left(s, x_{n}\left(g_{1}(s)\right), \ldots, x_{n}\left(g_{k}(s)\right)\right) \\
& \int_{\mu}^{+\infty} \frac{s^{n-2}}{|a(\mu)|}\left|f\left(s, x_{m}\left(g_{1}(s)\right), \ldots, x_{m}\left(g_{k}(s)\right)\right)-f\left(s, x\left(g_{1}(s)\right), \ldots, x\left(g_{k}(s)\right)\right)\right| d s \\
& \leqslant
\end{aligned}
$$

and

$$
\left|f\left(s, x_{m}\left(g_{1}(s)\right), \ldots, x_{m}\left(g_{k}(s)\right)\right)-f\left(s, x\left(g_{1}(s)\right), \ldots, x\left(g_{k}(s)\right)\right)\right| \rightarrow 0 \quad \text { as } m \rightarrow \infty, \quad s \in[\beta,+\infty),
$$

which together with the Lebesgue dominated convergence theorem yield that

$$
\lim _{m \rightarrow \infty}\left\|S_{2 L} x_{m}-S_{2 L} x\right\|=0,
$$


that is, $S_{2 L}$ is continuous in $\overline{\mathrm{U}(\mathrm{M})}$.

By virtue of (3.1), (3.19), (3.21), and (3.23), we get that for all $x \in \overline{\mathrm{U}(M)}$

$$
\begin{aligned}
\left\|S_{2 L} x\right\| & =\sup _{t \geqslant \beta}\left|\frac{(-1)^{n+1}}{b\left(\tau^{-1}(t)\right)(n-2) !} \int_{\tau^{-1}(t)}^{+\infty} \int_{\mu}^{+\infty} \frac{(s-\mu)^{n-2}}{a(\mu)}\left[f\left(s, x\left(g_{1}(s)\right), \ldots, x\left(g_{k}(s)\right)\right)-c(s)\right] d s d \mu\right| \\
& \leqslant \frac{1}{b_{*}(n-2) !} \int_{\tau^{-1}(T)}^{+\infty} \int_{\mu}^{+\infty} \frac{s^{n-2}}{|a(\mu)|}[p(s)+|c(s)|] d s d \mu \\
& \leqslant \frac{1}{b_{*}(n-2) !} \int_{\beta}^{+\infty} \int_{\mu}^{+\infty} \frac{s^{n-2}}{|a(\mu)|}[p(s)+|c(s)|] d s d \mu \\
& \leqslant \frac{1}{b_{*}}\left(\min \left\{b_{*} M-L, \frac{b_{*} L}{b^{*}}-M-b_{*} N\right\}-\varepsilon^{*}\right)<M,
\end{aligned}
$$

which gives that $S_{2 L}(\overline{\mathrm{U}(M)})$ is uniformly bounded in $[\beta,+\infty)$.

Let $\varepsilon>0$. It follows from (3.2) that there exist two constants $T_{1}$ and $T_{2}$ with $\min \left\{\tau^{-1}\left(T_{2}\right), T_{2}\right\}>T_{1}>$ $\max \{\mathrm{T}, \tau(\mathrm{T})\}$ satisfying

$$
\frac{1}{(n-2) !} \int_{T_{1}}^{+\infty} \int_{\mu}^{+\infty} \frac{s^{n-2}}{|a(\mu)|}[p(s)+|c(s)|] d s d \mu<\frac{b_{*} \varepsilon}{2}
$$

which together with (3.1) and (3.21) yields that for all $x \in \overline{\mathrm{U}(M)}$ and $t_{2}>t_{1} \geqslant T_{2}$

$$
\begin{aligned}
& \left|\left(S_{2 L} x\right)\left(t_{2}\right)-\left(S_{2 L} x\right)\left(t_{1}\right)\right|=\frac{1}{(n-2) !} \mid \frac{1}{b\left(\tau^{-1}\left(t_{2}\right)\right)} \int_{\tau^{-1}\left(t_{2}\right)}^{+\infty} \int_{\mu}^{+\infty} \frac{(s-\mu)^{n-2}}{a(\mu)}\left[f\left(s, x\left(g_{1}(s)\right), \ldots, x\left(g_{k}(s)\right)\right)\right. \\
& -c(s)] d s d \mu \\
& -\frac{1}{b\left(\tau^{-1}\left(t_{1}\right)\right)} \int_{\tau^{-1}\left(t_{1}\right)}^{+\infty} \int_{\mu}^{+\infty} \frac{(s-\mu)^{n-2}}{a(\mu)}\left[f\left(s, x\left(g_{1}(s)\right), \ldots, x\left(g_{k}(s)\right)\right)-c(s)\right] d s d \mu \mid \\
& \leqslant \frac{1}{b_{*}(n-2) !}\left[\int_{\tau^{-1}\left(t_{2}\right)}^{+\infty} \int_{\mu}^{+\infty} \frac{s^{n-2}}{|a(\mu)|}[p(s)+|c(s)|] d s d \mu\right. \\
& \left.+\int_{\tau^{-1}\left(t_{1}\right)}^{+\infty} \int_{\mu}^{+\infty} \frac{s^{n-2}}{|\mathfrak{a}(\mu)|}[\mathfrak{p}(\mathrm{s})+|\mathfrak{c}(\mathrm{s})|] \mathrm{d} s \mathrm{~d} \mu\right] \\
& \leqslant \frac{2}{b_{*}(n-2) !} \int_{\tau^{-1}\left(T_{2}\right)}^{+\infty} \int_{\mu}^{+\infty} \frac{s^{n-2}}{|a(\mu)|}[p(s)+|c(s)|] d s d \mu \\
& \leqslant \frac{2}{b_{*}(n-2) !} \int_{T_{1}}^{+\infty} \int_{\mu}^{+\infty} \frac{s^{n-2}}{|a(\mu)|}[p(s)+|c(s)|] d s d \mu<\varepsilon ;
\end{aligned}
$$

In view of (C2), (C3), and (3.2), we conclude that there exist positive constants $A_{1}$ and $M_{2}$ satisfying

$$
A_{1}=\min \left\{|a(t)|: t \in\left[\tau^{-1}(T), \tau^{-1}\left(T_{2}\right)\right]\right\}
$$

and

$$
M_{2}=\frac{1}{(n-2) !}\left[\frac{1}{A_{1} b_{*}} \int_{\tau^{-1}(T)}^{+\infty} s^{n-2}[p(s)+|c(s)|] d s+\frac{1}{b_{*}^{2}} \int_{\tau^{-1}(T)}^{+\infty} \int_{\mu}^{+\infty} \frac{s^{n-2}}{|a(\mu)|}[p(s)+|c(s)|] d s d \mu\right] .
$$

According to (C2), we infer that $\tau^{-1}$ and $b\left(\tau^{-1}\right)$ are continuous on $\left[T, T_{2}\right]$, which mean that there exists $\delta>0$ satisfying

$$
\sup \left\{\left|b\left(\tau^{-1}\left(t_{1}\right)\right)-b\left(\tau^{-1}\left(t_{2}\right)\right)\right|,\left|\tau^{-1}\left(t_{1}\right)-\tau^{-1}\left(t_{2}\right)\right| t_{1}, t_{2} \in\left[T, T_{2}\right],\left|t_{1}-t_{2}\right|<\delta\right\}<\frac{\varepsilon}{M_{2}}
$$


By (3.1), (3.23), (3.25), and (3.26), we obtain that for all $t_{1}, t_{2} \in\left[T, T_{2}\right]$ with $\left|t_{1}-t_{2}\right|<\delta$

$$
\begin{aligned}
& \left|\left(S_{2 L} x\right)\left(t_{2}\right)-\left(S_{2 L} x\right)\left(t_{1}\right)\right| \\
& =\frac{1}{(n-2) !} \mid \frac{1}{b\left(\tau^{-1}\left(t_{2}\right)\right)} \int_{\tau^{-1}\left(t_{2}\right)}^{+\infty} \int_{\mu}^{+\infty} \frac{(s-\mu)^{n-2}}{a(\mu)}\left[f\left(s, x\left(g_{1}(s)\right), \ldots, x\left(g_{k}(s)\right)\right)-c(s)\right] d s d \mu \\
& -\frac{1}{b\left(\tau^{-1}\left(t_{1}\right)\right)} \int_{\tau^{-1}\left(t_{1}\right)}^{+\infty} \int_{\mu}^{+\infty} \frac{(s-\mu)^{n-2}}{a(\mu)}\left[f\left(s, x\left(g_{1}(s)\right), \ldots, x\left(g_{k}(s)\right)\right)-c(s)\right] d s d \mu \mid \\
& =\frac{1}{(n-2) !} \mid \frac{1}{b\left(\tau^{-1}\left(t_{2}\right)\right)} \int_{\tau^{-1}\left(t_{2}\right)}^{+\infty} \int_{\mu}^{+\infty} \frac{(s-\mu)^{n-2}}{a(\mu)}\left[f\left(s, \chi\left(g_{1}(s)\right), \ldots, \chi\left(g_{k}(s)\right)\right)-c(s)\right] d s d \mu \\
& -\frac{1}{b\left(\tau^{-1}\left(t_{2}\right)\right)} \int_{\tau^{-1}\left(t_{1}\right)}^{+\infty} \int_{\mu}^{+\infty} \frac{(s-\mu)^{n-2}}{a(\mu)}\left[f\left(s, x\left(g_{1}(s)\right), \ldots, x\left(g_{k}(s)\right)\right)-c(s)\right] d s d \mu \\
& +\frac{1}{b\left(\tau^{-1}\left(t_{2}\right)\right)} \int_{\tau^{-1}\left(t_{1}\right)}^{+\infty} \int_{\mu}^{+\infty} \frac{(s-\mu)^{n-2}}{a(\mu)}\left[f\left(s, x\left(g_{1}(s)\right), \ldots, x\left(g_{k}(s)\right)\right)-c(s)\right] d s d \mu \\
& -\frac{1}{b\left(\tau^{-1}\left(t_{1}\right)\right)} \int_{\tau^{-1}\left(t_{1}\right)}^{+\infty} \int_{\mu}^{+\infty} \frac{(s-\mu)^{n-2}}{a(\mu)}\left[f\left(s, x\left(g_{1}(s)\right), \ldots, x\left(g_{k}(s)\right)\right)-c(s)\right] d s d \mu \mid \\
& \leqslant \frac{1}{(n-2) !}\left[\frac{1}{b\left(\tau^{-1}\left(t_{2}\right)\right)}\left|\int_{\tau^{-1}\left(t_{1}\right)}^{\tau^{-1}\left(t_{2}\right)} \int_{\mu}^{+\infty} \frac{(s-\mu)^{n-2}}{a(\mu)}\left[f\left(s, x\left(g_{1}(s)\right), \ldots, x\left(g_{k}(s)\right)\right)-c(s)\right] d s d \mu\right|\right. \\
& +\left|\frac{1}{b\left(\tau^{-1}\left(t_{2}\right)\right)}-\frac{1}{b\left(\tau^{-1}\left(t_{1}\right)\right)}\right| \int_{\tau^{-1}\left(t_{1}\right)}^{+\infty} \int_{\mu}^{+\infty} \frac{(s-\mu)^{n-2}}{|a(\mu)|} \\
& \left.\times\left|f\left(s, x\left(g_{1}(s)\right), \ldots, x\left(g_{k}(s)\right)\right)-c(s)\right| d s d \mu\right] \\
& \leqslant \frac{1}{b_{*}(n-2) !}\left[\frac{1}{A_{1}} \int_{\tau^{-1}(T)}^{+\infty} s^{n-2}[p(s)+|c(s)|] d s\left|\tau^{-1}\left(t_{1}\right)-\tau^{-1}\left(t_{2}\right)\right|\right. \\
& \left.+\frac{1}{b_{*}} \int_{\tau^{-1}(\mathrm{~T})}^{+\infty} \int_{\mu}^{+\infty} \frac{s^{n-2}}{|a(\mu)|}[p(s)+|c(s)|] d s d \mu\left|b\left(\tau^{-1}\left(t_{1}\right)\right)-b\left(\tau^{-1}\left(t_{2}\right)\right)\right|\right] \\
& \leqslant \frac{1}{(n-2) !}\left[\frac{1}{A_{1} b_{*}} \int_{\tau^{-1}(T)}^{+\infty} s^{n-2}[p(s)+|c(s)|] d s+\frac{1}{b_{*}^{2}} \int_{\tau^{-1}(T)}^{+\infty} \int_{\mu}^{+\infty} \frac{s^{n-2}}{|a(\mu)|}[p(s)+|c(s)|] d s d \mu\right] \\
& \times \sup \left\{\left|b\left(\tau^{-1}\left(t_{1}\right)\right)-b\left(\tau^{-1}\left(t_{2}\right)\right)\right|,\left|\tau^{-1}\left(t_{1}\right)-\tau^{-1}\left(t_{2}\right)\right| t_{1}, t_{2} \in\left[T, T_{2}\right],\left|t_{1}-t_{2}\right|<\delta\right\} \\
& <M_{2} \cdot \frac{\varepsilon}{M_{2}}=\varepsilon \text {. }
\end{aligned}
$$

By means of (3.23), we infer that for all $x \in \overline{\mathrm{U}(M)}$ and $t_{1}, t_{2} \in[\beta, T]$

$$
\left|\left(S_{2 L} x\right)\left(t_{2}\right)-\left(S_{2 L} x\right)\left(t_{1}\right)\right|=\left|\left(S_{2 L} x\right)(T)-\left(S_{2 L} x\right)(T)\right|<\varepsilon .
$$

It follows from (3.24), (3.27), and (3.28) that $S_{2 L}(\overline{U(M)})$ is equicontinuous in $[\beta,+\infty)$. Hence $S_{2 L}(\overline{U(M)})$ is relatively compact.

It follows from (3.19) and (3.22) that for all $x, y \in \overline{\mathrm{U}(M)}$ and $t \geqslant T$

$$
\left|\left(S_{1 \mathrm{~L}} x\right)(t)-\left(S_{1 \mathrm{~L}} \mathrm{y}\right)(\mathrm{t})\right|=\frac{1}{\mathrm{~b}\left(\tau^{-1}(\mathrm{t})\right)}|x(\tau(\mathrm{t}))-\mathrm{y}(\tau(\mathrm{t}))| \leqslant \frac{1}{\mathrm{~b}_{*}}\|x-y\|,
$$

which gives that

$$
\left\|S_{1 \mathrm{~L}} x-S_{1 \mathrm{~L}} y\right\| \leqslant \frac{1}{b_{*}}\|x-y\|,
$$

that is, $S_{1 L}$ is a contraction mapping in $\overline{\mathrm{U}(M)}$ and $\left\|S_{1 L}(\overline{\mathrm{U}(M)})\right\| \leqslant \frac{2 M}{b_{*}}$. Hence $S_{L}$ is a continuous, condensing mapping and $S_{\mathrm{L}}(\overline{\mathrm{U}(\mathrm{M})})$ is bounded. 
Let $\mathrm{P}$ and $\mathrm{Q}$ are defined by (3.13) and (3.14). It is clear that $\partial \mathrm{U}(\mathrm{M})=\mathrm{P} \cup \mathrm{Q}$. Suppose that there exist $x \in \partial U(M)$ and $\lambda \in(0,1)$ satisfying (3.15). Now we consider two possible cases as follows:

Case 1. Let $x \in$ P. It follows from (3.1), (3.13), (3.15), (3.19), and (3.21)-(3.23) that

$$
\begin{aligned}
x(t)= & (1-\lambda) p^{*}+\lambda S_{L} x(t) \\
= & (1-\lambda) p^{*}+\lambda\left[\frac{L}{b\left(\tau^{-1}(t)\right)}-\frac{x\left(\tau^{-1}(t)\right)}{b\left(\tau^{-1}(t)\right)}+\frac{(-1)^{n+1}}{b\left(\tau^{-1}(t)\right)(n-2) !} \int_{\tau^{-1}(t)}^{+\infty} \int_{\mu}^{+\infty} \frac{(s-\mu)^{n-2}}{a(\mu)}\right. \\
& \left.\times\left[f\left(s, x\left(g_{1}(s)\right), \ldots, x\left(g_{k}(s)\right)\right)-c(s)\right] d s d \mu\right] \\
\leqslant & (1-\lambda)\left(M-\varepsilon^{*}\right)+\lambda\left[\frac{L}{b_{*}}-\frac{N}{b^{*}}+\frac{1}{b_{*}(n-2) !} \int_{\tau^{-1}(T)}^{+\infty} \int_{\mu}^{+\infty} \frac{s^{n-2}}{|a(\mu)|}[p(s)+|c(s)|] d s d \mu\right] \\
\leqslant & (1-\lambda)\left(M-\varepsilon^{*}\right)+\lambda\left[\frac{L}{b_{*}}-\frac{N}{b^{*}}+\frac{1}{b_{*}} \min \left\{b_{*} M-L, \frac{b_{*} L}{b^{*}}-M-b_{*} N\right\}-\frac{\varepsilon^{*}}{b_{*}}\right] \\
\leqslant & \max \left\{M-\varepsilon^{*}, M-\frac{N}{b^{*}}-\frac{\varepsilon^{*}}{b_{*}}\right\}, \quad t \geqslant T,
\end{aligned}
$$

which yields that

$$
M=\|x\|=\sup _{t \geqslant \beta}|x(t)| \leqslant \max \left\{M-\varepsilon^{*}, M-\frac{N}{b^{*}}-\frac{\varepsilon^{*}}{b_{*}}\right\}<M,
$$

which is a contradiction.

Case 2. Let $x \in$ Q. It follows from (3.7), (3.14), (3.15), (3.19), and (3.21)-(3.23) that

$$
\begin{aligned}
N= & x\left(t^{*}\right)=(1-\lambda) p^{*}+\lambda S_{L} x\left(t^{*}\right) \\
= & (1-\lambda) p^{*}+\lambda\left[\frac{L}{b\left(\tau^{-1}\left(t^{*}\right)\right)}-\frac{x\left(\tau^{-1}\left(t^{*}\right)\right)}{b\left(\tau^{-1}\left(t^{*}\right)\right)}+\frac{(-1)^{n+1}}{b\left(\tau^{-1}\left(t^{*}\right)\right)(n-2) !}\right. \\
& \left.\times \int_{\max \left\{\tau^{-1}\left(t^{*}\right), \tau^{-1}(T)\right\}}^{+\infty} \int_{\mu}^{+\infty} \frac{(s-\mu)^{n-2}}{a(\mu)}\left[f\left(s, x\left(g_{1}(s)\right), \ldots, x\left(g_{k}(s)\right)\right)-c(s)\right] d s d \mu\right] \\
\geqslant & (1-\lambda)\left(M-\varepsilon^{*}\right)+\lambda\left[\frac{L}{b^{*}}-\frac{M}{b_{*}}-\frac{1}{b_{*}(n-2) !}\right. \\
& \left.\times \int_{\max \left\{\tau^{-1}\left(t^{*}\right), \tau^{-1}(T)\right\}}^{+\infty} \int_{\mu}^{+\infty} \frac{(s-\mu)^{n-2}}{|a(\mu)|}[p(s)+|c(s)|] d s d \mu\right] \\
\geqslant & (1-\lambda)\left(M-\varepsilon^{*}\right)+\lambda\left[\frac{L}{b^{*}}-\frac{M}{b_{*}}-\frac{1}{b_{*}} \min \left\{b_{*} M-L, \frac{b_{*} L}{b^{*}}-M-b_{*} N\right\}+\frac{\varepsilon^{*}}{b_{*}}\right] \\
\geqslant & (1-\lambda)\left(M-\varepsilon^{*}\right)+\lambda\left(N+\frac{\varepsilon^{*}}{b_{*}}\right) \\
\geqslant & \min \left\{M-\varepsilon^{*}, N+\frac{\varepsilon^{*}}{b_{*}}\right\}=N+\frac{\varepsilon^{*}}{b_{*}},
\end{aligned}
$$

which is impossible. Thus it follows from Lemma 2.1 that there exists $x \in \overline{U(M)}$ satisfying $S_{L} x=$ $\mathrm{S}_{1 \mathrm{~L}} \mathrm{x}+\mathrm{S}_{2 \mathrm{~L}} \mathrm{x}=\mathrm{x}$, that is,

$$
\begin{aligned}
x(t)= & \frac{L}{b\left(\tau^{-1}(t)\right)}-\frac{x\left(\tau^{-1}(t)\right)}{b\left(\tau^{-1}(t)\right)}+\frac{(-1)^{n+1}}{b\left(\tau^{-1}(t)\right)(n-2) !} \int_{\tau^{-1}(t)}^{+\infty} \int_{\mu}^{+\infty} \frac{(s-\mu)^{n-2}}{a(\mu)} \\
& \times\left[f\left(s, x\left(g_{1}(s)\right), \ldots, x\left(g_{k}(s)\right)\right)-c(s)\right] d s d \mu, \quad t \geqslant \max \left\{\tau^{-1}(T), T\right\},
\end{aligned}
$$

which gives that

$$
\begin{aligned}
x(\tau(t))= & \frac{L}{b(t)}-\frac{x(t)}{b(t)}+\frac{(-1)^{n+1}}{b(t)(n-2) !} \int_{t}^{+\infty} \int_{\mu}^{+\infty} \frac{(s-\mu)^{n-2}}{a(\mu)} \\
& \times\left[f\left(s, x\left(g_{1}(s)\right), \ldots, x\left(g_{k}(s)\right)\right)-c(s)\right] d s d \mu, \quad t \geqslant \max \left\{\tau^{-1}(T), T\right\}
\end{aligned}
$$


and

$$
\begin{aligned}
x(t)+b(t) x(\tau(t))= & L+\frac{(-1)^{n+1}}{(n-2) !} \int_{t}^{+\infty} \int_{\mu}^{+\infty} \frac{(s-\mu)^{n-2}}{a(\mu)} \\
& \times\left[f\left(s, x\left(g_{1}(s)\right), \ldots, x\left(g_{k}(s)\right)\right)-c(s)\right] d s d \mu, \quad t \geqslant \max \left\{\tau^{-1}(T), T\right\},
\end{aligned}
$$

which means that

$$
\left[a(t)(x(t)+b(t) x(\tau(t)))^{\prime}\right]^{(n-1)}=(-1)\left[f\left(t, x\left(g_{1}(t)\right), \ldots, x\left(g_{k}(t)\right)\right)-c(t)\right], \quad t \geqslant \max \left\{\tau^{-1}(T), T\right\},
$$

that is, $x$ is a bounded positive solution of Eq. (1.6) in $\overline{\mathrm{U}(\mathrm{M})}$.

Lastly we show that Eq. (1.6) has uncountably many bounded positive solutions in $\overline{\mathrm{U}(\mathrm{M})}$. Let $\mathrm{L}_{1}, \mathrm{~L}_{2} \in$ $\left(\frac{b^{*}}{b_{*}} M+b^{*} N, b_{*} M\right)$ and $L_{1} \neq L_{2}$. Analogously, we deduce that for each $\theta \in\{1,2\}$, there exist constants $\mathrm{T}_{\mathrm{L}_{\theta}}, \beta_{\theta}$ and a mapping $S_{\mathrm{L}_{\theta}}: \overline{\mathrm{U}(M)} \rightarrow \mathrm{V}(\mathrm{N})$ satisfying (3.7) and (3.19)-(3.23), where $\mathrm{L}, \beta, T, S_{1 L}, S_{2 L}$, and $S_{L}$ are replaced by $L_{\theta}, \beta_{\theta}, T_{L_{\theta}}, S_{1 L_{\theta}}, S_{2 L_{\theta}}$, and $S_{L_{\theta}}$, respectively, and the mapping $S_{L_{\theta}}$ has a fixed point $\chi^{\theta} \in \overline{\mathrm{U}(M)}$, which is a bounded positive solution of Eq. (1.6) in $\overline{\mathrm{U}(M)}$, that is,

$$
\begin{aligned}
x^{\theta}(t)= & \frac{L_{\theta}}{b\left(\tau^{-1}(t)\right)}-\frac{x^{\theta}\left(\tau^{-1}(t)\right)}{b\left(\tau^{-1}(t)\right)}+\frac{(-1)^{n+1}}{b\left(\tau^{-1}(t)\right)(n-2) !} \int_{\tau^{-1}(t)}^{+\infty} \int_{\mu}^{+\infty} \frac{(s-\mu)^{n-2}}{a(\mu)} \\
& \times\left[f\left(s, x^{\theta}\left(g_{1}(s)\right), \ldots, x^{\theta}\left(g_{k}(s)\right)\right)-c(s)\right] d s d \mu, \quad t \geqslant T_{L_{\theta}} .
\end{aligned}
$$

It follows from (3.2) that there exists a constant $T_{*}$ with $\min \left\{T_{*}, \tau^{-1}\left(T_{*}\right)\right\}>\max \left\{T_{L_{1}}, T_{L_{2}}\right\}$ satisfying

$$
\frac{1}{(n-2) !} \int_{\tau^{-1}\left(T_{*}\right)}^{+\infty} \int_{\mu}^{+\infty} \frac{s^{n-2}}{|a(\mu)|} p(s) d s d \mu<\frac{b_{*}\left|L_{1}-L_{2}\right|}{4 b^{*}} \text {. }
$$

In order to prove that the set of bounded positive solutions of Eq. (1.6) is uncountable, it is sufficient to prove that $x^{1} \neq x^{2}$. From (3.1), (3.29), and (3.30), we get that for $t \geqslant T_{*}$

$$
\begin{aligned}
\left|x^{1}(t)-x^{2}(t)\right|= & \mid \frac{L_{1}}{b\left(\tau^{-1}(t)\right)}-\frac{L_{2}}{b\left(\tau^{-1}(t)\right)}-\frac{x^{1}\left(\tau^{-1}(t)\right)}{b\left(\tau^{-1}(t)\right)}+\frac{x^{2}\left(\tau^{-1}(t)\right)}{b\left(\tau^{-1}(t)\right)}+\frac{(-1)^{n+1}}{b\left(\tau^{-1}(t)\right)(n-2) !} \\
& \times \int_{\tau^{-1}(t)}^{+\infty} \int_{\mu}^{+\infty} \frac{(s-\mu)^{n-2}}{a(\mu)}\left[f\left(s, x^{1}\left(g_{1}(s)\right), \ldots, x^{1}\left(g_{k}(s)\right)\right)\right. \\
& \left.-f\left(s, x^{2}\left(g_{1}(s)\right), \ldots, x^{2}\left(g_{k}(s)\right)\right)\right] d s d \mu \mid \\
\geqslant & \frac{1}{b^{*}}\left|L_{1}-L_{2}\right|-\frac{1}{b_{*}}\left|x^{1}\left(\tau^{-1}(t)\right)-x^{2}\left(\tau^{-1}(t)\right)\right|-\frac{1}{b_{*}(n-2) !} \int_{\tau^{-1}(t)}^{+\infty} \int_{\mu}^{+\infty} \frac{(s-\mu)^{n-2}}{|a(\mu)|} \\
& \times\left[\left|f\left(s, x^{1}\left(g_{1}(s)\right), \ldots, x^{1}\left(g_{k}(s)\right)\right)\right|+\left|f\left(s, x^{2}\left(g_{1}(s)\right), \ldots, x^{2}\left(g_{k}(s)\right)\right)\right|\right] d s d \mu \\
\geqslant & \frac{1}{b^{*}}\left|L_{1}-L_{2}\right|-\frac{1}{b_{*}}\left\|x^{1}-x^{2}\right\|-\frac{2}{b_{*}(n-2) !} \int_{\tau^{-1}\left(T_{*}\right)}^{+\infty} \int_{\mu}^{+\infty} \frac{s^{n-2}}{|a(\mu)|} p(s) d s d \mu \\
> & \frac{1}{2 b^{*}}\left|L_{1}-L_{2}\right|-\frac{1}{b_{*}}\left\|x^{1}-x^{2}\right\|,
\end{aligned}
$$

which means that

$$
\left\|x^{1}-x^{2}\right\|>\frac{b_{*}\left|L_{1}-L_{2}\right|}{2 b^{*}\left(1+b_{*}\right)}>0,
$$

that is, $x^{1} \neq x^{2}$. This completes the proof.

Theorem 3.3. Let (C1), (C2), and (C3) hold. Assume that there exist four constants $\mathrm{N}, \mathrm{M}, \mathrm{b}_{*}$ and $\mathrm{b}^{*}$ and $a$ function $\mathrm{p} \in \mathrm{C}\left(\left[\mathrm{t}_{0},+\infty\right), \mathbb{R}^{+}\right)$satisfying (3.1), (3.2), and

$$
\left(1+\mathrm{b}^{*}\right) \mathrm{M}<\left(1+\mathrm{b}_{*}\right) \mathrm{N}<0, \quad \mathrm{~b}_{*} \leqslant \mathrm{~b}(\mathrm{t}) \leqslant \mathrm{b}^{*}<-1, \text { eventually. }
$$

Then Eq. (1.6) has uncountably many bounded positive solutions. 
Proof. Let $\mathrm{L} \in\left(\left(1+\mathrm{b}^{*}\right) \mathrm{M},\left(1+\mathrm{b}_{*}\right) \mathrm{N}\right)$. Now we show that there exists a mapping $\mathrm{S}_{\mathrm{L}}: \overline{\mathrm{U}(M)} \rightarrow \mathrm{V}(\mathrm{N})$ such that it has a fixed point $x \in \overline{\mathrm{U}(\mathrm{M})}$, which is also a bounded positive solution of Eq. (1.6). (C2) and (C3) mean that $\tau^{-1}$ is strictly increasing and continuous in $\left[\tau\left(t_{0}\right),+\infty\right)$ and $\lim _{t \rightarrow+\infty} \tau^{-1}(t)=+\infty$. It follows from (3.2) and (3.31) that there exist two constants $\beta$ and T satisfying

$$
\begin{aligned}
& \min \left\{T, \tau^{-1}(T)\right\}>\beta \geqslant \max \left\{1,\left|t_{0}\right|+\left|\beta_{0}\right|,\left|\tau\left(t_{0}\right)\right|\right\}, b_{*} \leqslant b(t) \leqslant b^{*}<-1, \quad t \geqslant \beta, \\
& \frac{1}{(n-2) !} \int_{\beta}^{+\infty} \int_{\mu}^{+\infty} \frac{s^{n-2}}{|a(\mu)|}[p(s)+|c(s)|] d s d \mu<\min \left\{L-\left(1+b^{*}\right) M, \frac{b^{*}}{b_{*}}\left[N\left(1+b_{*}\right)-L\right]\right\} .
\end{aligned}
$$

Let $\mathrm{p}^{*}=M-\varepsilon^{*}$, where $\varepsilon^{*} \in\left(0, \min \left\{L-\left(1+\mathrm{b}^{*}\right) M, \frac{\mathrm{b}^{*}}{\mathrm{~b}_{*}}\left[N\left(1+\mathrm{b}_{*}\right)-\mathrm{L}, \frac{\mathrm{b}^{*}(M-N)}{\mathrm{b}^{*}-1}\right]\right\}\right)$ is enough small and

$$
\frac{1}{(n-2) !} \int_{\beta}^{+\infty} \int_{\mu}^{+\infty} \frac{s^{n-2}}{|a(\mu)|}[p(s)+|c(s)|] d s d \mu<\min \left\{L-\left(1+b^{*}\right) M, \frac{b^{*}}{b_{*}}\left[N\left(1+b_{*}\right)-L\right]\right\}-\varepsilon^{*} .
$$

Obviously, $p^{*} \in U(N, M)$. Define a mapping $S_{L}: \bar{U}(M) \rightarrow C B([\beta,+\infty), \mathbb{R})$ by (3.7), where the mappings $\mathrm{S}_{1 \mathrm{~L}}, \mathrm{~S}_{2 \mathrm{~L}}: \overline{\mathrm{U}(\mathrm{M})} \rightarrow \mathrm{CB}([\beta,+\infty), \mathbb{R})$ are defined by (3.22) and (3.23), respectively.

By virtue of (3.1), (3.7), (3.22), (3.23), (3.32), and (3.33), we get that for any $x \in \overline{\mathrm{U}(M)}$ and $t \geqslant T$

$$
\begin{aligned}
\left(S_{L}\right)(x)= & \left(S_{1 L} x\right)(t)+\left(S_{2 L} x\right)(t) \\
= & \frac{L}{b\left(\tau^{-1}(t)\right)}-\frac{x\left(\tau^{-1}(t)\right)}{b\left(\tau^{-1}(t)\right)}+\frac{(-1)^{n+1}}{b\left(\tau^{-1}(t)\right)(n-2) !} \int_{\tau^{-1}(t)}^{+\infty} \int_{\mu}^{+\infty} \frac{(s-\mu)^{n-2}}{a(\mu)} \\
& \times\left[f\left(s, x\left(g_{1}(s)\right), \ldots, x\left(g_{k}(s)\right)\right)-c(s)\right] d s d \mu \\
\geqslant & \frac{L}{b_{*}}-\frac{N}{b_{*}}+\frac{1}{b^{*}(n-2) !} \int_{\tau^{-1}(T)}^{+\infty} \int_{\mu}^{+\infty} \frac{s^{n-2}}{|a(\mu)|}[p(s)+|c(s)|] d s d \mu \\
\geqslant & \frac{L}{b_{*}}-\frac{N}{b_{*}}+\frac{1}{b^{*}(n-2) !} \int_{\beta}^{+\infty} \int_{\mu}^{+\infty} \frac{s^{n-2}}{|a(\mu)|}[p(s)+|c(s)|] d s d \mu \\
\geqslant & \frac{L}{b_{*}}-\frac{N}{b_{*}}+\frac{1}{b^{*}} \min \left\{L-\left(1+b^{*}\right) M, \frac{b^{*}}{b_{*}}\left[N\left(1+b_{*}\right)-L\right]\right\}-\frac{1}{b^{*}} \varepsilon^{*} \\
\geqslant & N-\frac{1}{b^{*}} \varepsilon^{*}>N,
\end{aligned}
$$

which gives that $S_{L}(\overline{U(M)}) \subseteq V(N)$. The rest of the proof is similar to that of Theorems 3.2, and is omitted. This completes the proof.

Next we employ the Rothe fixed point theorem to prove the existence and multiplicity of bounded positive solutions of Eq. (1.6).

Theorem 3.4. Let (C1), (C2), (C3), and (C4) hold. Assume that there exist two constants $\mathrm{M}$ and $\mathrm{N}$ with $\mathrm{M}>$ $\mathrm{N}>0$ and a function $\mathrm{p} \in \mathrm{C}\left(\left[\mathrm{t}_{0},+\infty\right), \mathbb{R}^{+}\right)$satisfying (3.1), (3.2), and

$$
\mathrm{b}(\mathrm{t})=1 \text {, eventually. }
$$

Then Eq. (1.6) has uncountably many bounded positive solutions.

Proof. Let $L \in(M-N, M+N)$. First of all we prove that there exists a mapping $S_{L}: \overline{B(M, N)} \rightarrow$ $\mathrm{CB}([\beta,+\infty), \mathbb{R})$ with $S_{\mathrm{L}}(\partial \mathrm{B}(M, N)) \subseteq \overline{\mathrm{B}(M, N)}$ such that $S_{\mathrm{L}}$ has a fixed point $x \in \overline{\mathrm{B}(M, N)}$, which is also a bounded positive solution of Eq. (1.6). It follows from (3.2), (3.34), (C3), and (C4) that there exist two constants $\mathrm{T}$ and $\beta$ satisfying

$$
\tau^{-1}(T)>T>\beta \geqslant \max \left\{1,\left|t_{0}\right|+\left|\beta_{0}\right|,\left|\tau\left(t_{0}\right)\right|\right\}, \quad b(t)=1, \quad t \geqslant \beta ;
$$




$$
\frac{2}{(n-2) !} \int_{\beta}^{+\infty} \int_{\mu}^{+\infty} \frac{s^{n-2}}{|a(\mu)|}[p(s)+|c(s)|] d s d \mu<\min \{M+N-L, N-M+L\} .
$$

Define a mapping $S_{\mathrm{L}}: \overline{\mathrm{B}(\mathrm{M}, \mathrm{N})} \rightarrow \mathrm{CB}([\beta,+\infty), \mathbb{R})$ as follows:

$$
\left(S_{L} x\right)(t)= \begin{cases}L+\frac{(-1)^{n+1}}{(n-2) !} \sum_{j=1}^{\infty} \int_{\tau^{-2 j+1}(t)}^{\tau^{-2 j}(t)} \int_{\mu}^{+\infty} \frac{(s-\mu)^{n-2}}{a(\mu)}\left[f\left(s, x\left(g_{1}(s)\right), \ldots, x\left(g_{k}(s)\right)\right)-c(s)\right] d s d \mu, & t \geqslant T, \\ \left(S_{L} x\right)(T), & \beta \leqslant t<T,\end{cases}
$$

for each $x \in \overline{\mathrm{B}(\mathrm{M}, \mathrm{N})}$. It follows from (3.1), (3.13), (3.14), (3.27), (3.28), and Lemma 2.3 that for any $x \in \partial \mathrm{B}(\mathrm{M}, \mathrm{N}) \subseteq \overline{\mathrm{B}(\mathrm{M}, \mathrm{N})}$ and $\mathrm{t} \geqslant \mathrm{T}$

$$
\begin{aligned}
\left|\left(S_{L} x\right)(t)-M\right| & =\left|L-M+\frac{(-1)^{n+1}}{(n-2) !} \sum_{j=1}^{\infty} \int_{\tau^{-2 j+1}(t)}^{\tau^{-2 j}(t)} \int_{\mu}^{+\infty} \frac{(s-\mu)^{n-2}}{a(\mu)}\left[f\left(s, x\left(g_{1}(s)\right), \ldots, x\left(g_{k}(s)\right)\right)-c(s)\right] d s d \mu\right| \\
& \leqslant|L-M|+\frac{1}{(n-2) !} \sum_{j=1}^{\infty} \int_{\tau^{-2 j+1}(t)}^{\tau^{-2 j}(t)} \int_{\mu}^{+\infty} \frac{s^{n-2}}{|a(\mu)|}[p(s)+|c(s)|] d s d \mu \\
& \leqslant|L-M|+\frac{1}{(n-2) !} \int_{\tau^{-1}(T)}^{+\infty} \int_{\mu}^{+\infty} \frac{s^{n-2}}{|a(\mu)|}[p(s)+|c(s)|] d s d \mu \\
& \leqslant|L-M|+\frac{1}{(n-2) !} \int_{\beta}^{+\infty} \int_{\mu}^{+\infty} \frac{s^{n-2}}{|a(\mu)|}[p(s)+|c(s)|] d s d \mu \\
& <|L-M|+\frac{1}{2} \min \{M+N-L, N-M+L\}<N,
\end{aligned}
$$

which yields that $S_{L}(\partial B(M, N)) \subseteq \overline{B(M, N)}$.

Now we assert that $S_{L}$ is a continuous, condensing mapping in $\overline{B(M, N)}$. Let $\left\{x_{m}\right\}_{m} \in \mathbb{N} \subseteq \overline{B(M, N)}$ and $x \in \overline{\mathrm{B}(M, N)}$ with $\lim _{m \rightarrow \infty} x_{m}=x$. By virtue of (3.1), (3.36), (3.37), and the continuity of $f$ and Lemma 2.3, we infer that for each $m \in \mathbb{N}$

$$
\begin{aligned}
&\left\|S_{L} x_{m}-S_{L} x\right\|= \sup _{t \geqslant T} \mid \frac{(-1)^{n+1}}{(n-2) !} \sum_{j=1}^{\infty} \int_{\tau^{-2 j+1}(t)}^{\tau^{-2 j}(t)} \int_{\mu}^{+\infty} \frac{(s-\mu)^{n-2}}{a(\mu)}\left[f\left(s, x_{m}\left(g_{1}(s)\right), \ldots, x_{m}\left(g_{k}(s)\right)\right)\right. \\
&\left.-f\left(s, x\left(g_{1}(s)\right), \ldots, x\left(g_{k}(s)\right)\right)\right] d s d \mu \mid \\
& \leqslant \frac{1}{(n-2) !} \int_{\tau^{-1}(T)}^{+\infty} \int_{\mu}^{+\infty} \frac{s^{n-2}}{|a(\mu)|} \mid f\left(s, x_{m}\left(g_{1}(s)\right), \ldots, x_{m}\left(g_{k}(s)\right)\right) \\
&-f\left(s, x\left(g_{1}(s)\right), \ldots, x\left(g_{k}(s)\right)\right) \mid d s d \mu \\
& \leqslant \frac{1}{(n-2) !} \int_{\beta}^{+\infty} \int_{\mu}^{+\infty} \frac{s^{n-2}}{|a(\mu)|} \mid f\left(s, x_{m}\left(g_{1}(s)\right), \ldots, x_{m}\left(g_{k}(s)\right)\right) \\
&-f\left(s, x\left(g_{1}(s)\right), \ldots, x\left(g_{k}(s)\right)\right) \mid d s d \mu, \\
& \int_{\mu}^{+\infty} \frac{s^{n-2}}{|a(\mu)|}\left|f\left(s, x_{m}\left(g_{1}(s)\right), \ldots, x_{m}\left(g_{k}(s)\right)\right)-f\left(s, x\left(g_{1}(s)\right), \ldots, x\left(g_{k}(s)\right)\right)\right| d s \\
& \leqslant 2 \int_{\mu}^{+\infty} \frac{s^{n-2}}{|a(\mu)|} p(s) d s, \quad \mu \in[\beta,+\infty),
\end{aligned}
$$

and

$$
\left|f\left(s, x_{m}\left(g_{1}(s)\right), \ldots, x_{m}\left(g_{k}(s)\right)\right)-f\left(s, x\left(g_{1}(s)\right), \ldots, x\left(g_{k}(s)\right)\right)\right| \rightarrow 0 \quad \text { as } m \rightarrow \infty, \quad s \in[\beta,+\infty),
$$


which together with the Lebesgue dominated convergence theorem give that

$$
\lim _{m \rightarrow \infty} S_{L} x_{m}=S_{L} x
$$

that is, $S_{\mathrm{L}}$ is continuous in $\overline{\mathrm{B}(\mathrm{M}, \mathrm{N})}$.

In light of (3.1), (3.36), (3.37), and Lemma 2.3, we get that for any $x \in \overline{\mathrm{B}(M, N)}$

$$
\begin{aligned}
\left\|S_{L} x\right\| & =\left|L+\frac{(-1)^{n+1}}{(n-2) !} \sum_{j=1}^{\infty} \int_{\tau^{-2 j+1}(t)}^{\tau^{-2 j}(t)} \int_{\mu}^{+\infty} \frac{(s-\mu)^{n-2}}{a(\mu)}\left[f\left(s, x\left(g_{1}(s)\right), \ldots, x\left(g_{k}(s)\right)\right)-c(s)\right] d s d \mu\right| \\
& \leqslant L+\frac{1}{(n-2) !} \int_{\tau^{-1}(T)}^{+\infty} \int_{\mu}^{+\infty} \frac{s^{n-2}}{|a(\mu)|}[p(s)+|c(s)|] d s d \mu \\
& \leqslant L+\frac{1}{(n-2) !} \int_{\beta}^{+\infty} \int_{\mu}^{+\infty} \frac{s^{n-2}}{|a(\mu)|}[p(s)+|c(s)|] d s d \mu \\
& <L+\frac{1}{2} \min \{M+N-L, N-M+L\} \leqslant \frac{M+N+L}{2}
\end{aligned}
$$

which implies that $S_{L}(\overline{B(M, N)})$ is uniformly bounded in $[\beta,+\infty)$.

Let $\varepsilon>0$. Notice that (3.2) ensures that there exists $T^{*}>\tau^{-1}(T)$ satisfying

$$
\frac{1}{(n-2) !} \int_{T^{*}}^{+\infty} \int_{\mu}^{+\infty} \frac{s^{n-2}}{|a(\mu)|}[p(s)+|c(s)|] d s d \mu<\frac{\varepsilon}{4},
$$

which together with (3.1), (3.37), and Lemma 2.3 yields that for all $x \in \overline{\mathrm{B}(\mathrm{M}, \mathrm{N})}$ and $t_{2}>t_{1} \geqslant \mathrm{~T}^{*}$

$$
\begin{aligned}
\left|\left(S_{L} x\right)\left(t_{2}\right)-\left(S_{L} x\right)\left(t_{1}\right)\right| & \mid \frac{(-1)^{n+1}}{(n-2) !} \sum_{j=1}^{\infty} \int_{\tau^{-2 j+1}\left(t_{2}\right)}^{\tau^{-2 j}\left(t_{2}\right)} \int_{\mu}^{+\infty} \frac{(s-\mu)^{n-2}}{a(\mu)}\left[f\left(s, x\left(g_{1}(s)\right), \ldots, x\left(g_{k}(s)\right)\right)-c(s)\right] d s d \mu \\
& -\frac{(-1)^{n+1}}{(n-2) !} \sum_{j=1}^{\infty} \int_{\tau^{-2 j+1}\left(t_{1}\right)}^{\tau^{-2 j}\left(t_{1}\right)} \int_{\mu}^{+\infty} \frac{(s-\mu)^{n-2}}{a(\mu)}\left[f\left(s, x\left(g_{1}(s)\right), \ldots, x\left(g_{k}(s)\right)\right)-c(s)\right] d s d \mu \mid \\
\leqslant & \frac{1}{(n-2) !}\left[\int_{\tau^{-1}\left(t_{2}\right)}^{+\infty} \int_{\mu}^{+\infty} \frac{s^{n-2}}{|a(\mu)|}[p(s)+|c(s)|] d s d \mu\right. \\
& \left.+\int_{\tau^{-1}\left(t_{1}\right)}^{+\infty} \int_{\mu}^{+\infty} \frac{s^{n-2}}{|a(\mu)|}[p(s)+|c(s)|] d s d \mu\right] \\
\leqslant & \frac{2}{(n-2) !} \int_{\tau^{-1}\left(T^{*}\right)}^{+\infty} \int_{\mu}^{+\infty} \frac{s^{n-2}}{|a(\mu)|}[p(s)+|c(s)|] d s d \mu \\
\leqslant & \frac{2}{(n-2) !} \int_{T^{*}}^{+\infty} \int_{\mu}^{+\infty} \frac{s^{n-2}}{|a(\mu)|}[p(s)+|c(s)|] d s d \mu<\varepsilon .
\end{aligned}
$$

Notice that (3.2), (C2)-(C4), and Lemma 2.3 guarantee that there exists $H \in \mathbb{N}$ satisfying $\tau^{-2 H+1}(T)>T^{*}$. Put

$$
A_{2}=\min \left\{|a(t)|: t \in\left[\tau^{-j}(T), \tau^{-j}\left(T^{*}\right)\right], 1 \leqslant j \leqslant 2 H\right\}
$$

and

$$
M_{3}=1+\frac{1}{(n-2) ! A_{2}} \int_{\tau^{-1}(T)}^{+\infty} s^{n-2}[p(s)+|c(s)|] d s
$$


Since $\tau^{-j}$ is uniformly continuous in $\left[T, T^{*}\right]$ for $1 \leqslant j \leqslant 2 H$, it follows that there exists $\delta>0$ satisfying

$$
\sup \left\{\left|\tau^{-j}\left(t_{1}\right)-\tau^{-j}\left(t_{2}\right)\right|: 1 \leqslant j \leqslant 2 H, t_{1}, t_{2} \in\left[T, T^{*}\right],\left|t_{2}-t_{1}\right|<\delta\right\}<\frac{\varepsilon}{4 \mathrm{HM}_{3}},
$$

which together with (3.1), (3.37), (3.38), and (3.40) that for all $t_{1}, t_{2} \in\left[T, T^{*}\right]$ with $t_{1} \leqslant t_{2}<t_{1}+\delta$ and all $x \in \overline{\mathrm{B}(\mathrm{M}, \mathrm{N})}$

$$
\begin{aligned}
& \left|\left(S_{L} x\right)\left(t_{2}\right)-\left(S_{L} x\right)\left(t_{1}\right)\right| \\
& =\mid \frac{(-1)^{n+1}}{(n-2) !} \sum_{j=1}^{\infty} \int_{\tau^{-2 j+1}\left(t_{2}\right)}^{\tau^{-2 j}\left(t_{2}\right)} \int_{\mu}^{+\infty} \frac{(s-\mu)^{n-2}}{a(\mu)}\left[f\left(s, x\left(g_{1}(s)\right), \ldots, x\left(g_{k}(s)\right)\right)-c(s)\right] d s d \mu \\
& -\frac{(-1)^{n+1}}{(n-2) !} \sum_{j=1}^{\infty} \int_{\tau^{-2 j+1}\left(t_{1}\right)}^{\tau^{-2 j}\left(t_{1}\right)} \int_{\mu}^{+\infty} \frac{(s-\mu)^{n-2}}{a(\mu)}\left[f\left(s, x\left(g_{1}(s)\right), \ldots, x\left(g_{k}(s)\right)\right)-c(s)\right] d s d \mu \\
& =\frac{1}{(n-2) !} \mid \sum_{j=1}^{H} \int_{\tau^{-2 j+1}\left(t_{2}\right)}^{\tau^{-2 j}\left(t_{2}\right)} \int_{\mu}^{+\infty} \frac{(s-\mu)^{n-2}}{a(\mu)}\left[f\left(s, x\left(g_{1}(s)\right), \ldots, x\left(g_{k}(s)\right)\right)-c(s)\right] d s d \mu \\
& -\sum_{j=1}^{H} \int_{\tau^{-2 j+1}\left(t_{1}\right)}^{\tau^{-2 j}\left(t_{1}\right)} \int_{\mu}^{+\infty} \frac{(s-\mu)^{n-2}}{a(\mu)}\left[f\left(s, x\left(g_{1}(s)\right), \ldots, x\left(g_{k}(s)\right)\right)-c(s)\right] d s d \mu \\
& +\sum_{j=H+1}^{\infty} \int_{\tau^{-2 j+1}\left(t_{2}\right)}^{\tau^{-2 j}\left(t_{2}\right)} \int_{\mu}^{+\infty} \frac{(s-\mu)^{n-2}}{a(\mu)}\left[f\left(s, x\left(g_{1}(s)\right), \ldots, \chi\left(g_{k}(s)\right)\right)-c(s)\right] d s d \mu \\
& -\sum_{j=H+1}^{\infty} \int_{\tau^{-2 j+1}\left(t_{1}\right)}^{\tau^{-2 j}\left(t_{1}\right)} \int_{\mu}^{+\infty} \frac{(s-\mu)^{n-2}}{a(\mu)}\left[f\left(s, x\left(g_{1}(s)\right), \ldots, \chi\left(g_{k}(s)\right)\right)-c(s)\right] d s d \mu \mid \\
& \leqslant \frac{1}{(n-2) !}\left\{\mid \sum_{j=1}^{H} \int_{\tau^{-2 j}\left(t_{1}\right)}^{\tau^{-2 j}\left(t_{2}\right)} \int_{\mu}^{+\infty} \frac{(s-\mu)^{n-2}}{a(\mu)}\left[f\left(s, x\left(g_{1}(s)\right), \ldots, x\left(g_{k}(s)\right)\right)-c(s)\right] d s d \mu\right. \\
& +\sum_{j=1}^{H} \int_{\tau^{-2 j+1}\left(t_{2}\right)}^{\tau^{-2 j}\left(t_{1}\right)} \int_{\mu}^{+\infty} \frac{(s-\mu)^{n-2}}{a(\mu)}\left[f\left(s, x\left(g_{1}(s)\right), \ldots, x\left(g_{k}(s)\right)\right)-c(s)\right] d s d \mu \\
& -\sum_{j=1}^{H} \int_{\tau^{-2 j+1}\left(t_{1}\right)}^{\tau^{-2 j}\left(t_{1}\right)} \int_{\mu}^{+\infty} \frac{(s-\mu)^{n-2}}{a(\mu)}\left[f\left(s, x\left(g_{1}(s)\right), \ldots, x\left(g_{k}(s)\right)\right)-c(s)\right] d s d \mu \mid \\
& +\sum_{j=H+1}^{\infty} \int_{\tau^{-2 j+1}\left(t_{2}\right)}^{\tau^{-2 j}\left(t_{2}\right)} \int_{\mu}^{+\infty} \frac{(s-\mu)^{n-2}}{|a(\mu)|}[p(s)+|c(s)|] d s d \mu \\
& \left.+\sum_{j=H+1}^{\infty} \int_{\tau^{-2 j+1}\left(t_{1}\right)}^{\tau^{-2 j}\left(t_{1}\right)} \int_{\mu}^{+\infty} \frac{(s-\mu)^{n-2}}{|a(\mu)|}[p(s)+|c(s)|] d s d \mu\right\} \\
& \leqslant \frac{1}{(n-2) !}\left\{\sum_{j=1}^{H} \int_{\tau^{-2 j}\left(t_{1}\right)}^{\tau^{-2 j}\left(t_{2}\right)} \int_{\mu}^{+\infty} \frac{s^{n-2}}{|a(\mu)|}[p(s)+|c(s)|] d s d \mu\right. \\
& +\sum_{j=1}^{H} \int_{\tau^{-2 j+1}\left(t_{1}\right)}^{\tau^{-2 j+1}\left(t_{2}\right)} \int_{\mu}^{+\infty} \frac{s^{n-2}}{|a(\mu)|}[p(s)+|c(s)|] d s d \mu \\
& \left.+\int_{\tau^{-2 H+1}\left(t_{2}\right)}^{+\infty} \int_{\mu}^{+\infty} \frac{s^{n-2}}{|a(\mu)|}[p(s)+|c(s)|] d s d \mu+\int_{\tau^{-2 H+1}\left(t_{1}\right)}^{+\infty} \int_{\mu}^{+\infty} \frac{s^{n-2}}{|a(\mu)|}[p(s)+|c(s)|] d s d \mu\right\} \\
& \leqslant \frac{1}{(n-2) !}\left\{\sum_{j=1}^{2 H} \int_{\tau^{-j}\left(t_{1}\right)}^{\tau^{-j}\left(t_{2}\right)} \int_{\mu}^{+\infty} \frac{s^{n-2}}{|a(\mu)|}[p(s)+|c(s)|] d s d \mu\right.
\end{aligned}
$$




$$
\begin{aligned}
& \left.+2 \int_{\mathrm{T}^{*}}^{+\infty} \int_{\mu}^{+\infty} \frac{\mathrm{s}^{\mathrm{n}-2}}{|\mathrm{a}(\mu)|}[\mathrm{p}(\mathrm{s})+|\mathrm{c}(\mathrm{s})|] \mathrm{dsd} \mu\right\} \\
\leqslant & 2 \mathrm{HM} M_{3} \sup \left\{\left|\tau^{-j}\left(\mathrm{t}_{1}\right)-\tau^{-j}\left(\mathrm{t}_{2}\right)\right|: 1 \leqslant j \leqslant 2 \mathrm{H}, \mathrm{t}_{1}, \mathrm{t}_{2} \in\left[\mathrm{T}, \mathrm{T}^{*}\right],\left|\mathrm{t}_{2}-\mathrm{t}_{1}\right|<\delta\right\}+\frac{\varepsilon}{2}<\varepsilon .
\end{aligned}
$$

In view of (3.37), we infer that for all $x \in \overline{\mathrm{B}(\mathrm{M}, \mathrm{N})}$ and $t_{1}, \mathrm{t}_{2} \in[\beta, T]$

$$
\left|\left(S_{L} x\right)\left(t_{2}\right)-\left(S_{L} x\right)\left(t_{1}\right)\right|=\left|\left(S_{L} x\right)(T)-\left(S_{L} x\right)(T)\right|<\varepsilon .
$$

It follows from (3.39), (3.41), and (3.42) that $S_{L}(\overline{\mathrm{U}(M)})$ is equicontinuous in $[\beta,+\infty)$. Hence $S_{L}(\overline{B(M, N)})$ is relatively compact, that is, $S_{L}$ is condensing in $\overline{B(M, N)}$.

Thus Lemma 2.2 ensures that there exists $x \in \overline{\mathrm{B}(\mathrm{M}, \mathrm{N})}$ such that $S_{\mathrm{L}} x=x$, which means that

$$
x(t)=L+\frac{(-1)^{n+1}}{(n-2) !} \sum_{j=1}^{\infty} \int_{\tau^{-2 j+1}(t)}^{\tau^{-2 j}(t)} \int_{\mu}^{+\infty} \frac{(s-\mu)^{n-2}}{a(\mu)}\left[f\left(s, x\left(g_{1}(s)\right), \ldots, x\left(g_{k}(s)\right)\right)-c(s)\right] d s d \mu, \quad t \geqslant \tau^{-1}(T),
$$

which together with Lemma 2.3 implies that

$$
\begin{aligned}
x(t)+x(\tau(t)) & =2 L+\frac{(-1)^{n+1}}{(n-2) !} \sum_{j=1}^{\infty} \int_{\tau^{-2 j+2}(t)}^{\tau^{-2 j}(t)} \int_{\mu}^{+\infty} \frac{(s-\mu)^{n-2}}{a(\mu)}\left[f\left(s, x\left(g_{1}(s)\right), \ldots, x\left(g_{k}(s)\right)\right)-c(s)\right] d s d \mu \\
& =2 L+\frac{(-1)^{n+1}}{(n-2) !} \int_{t}^{+\infty} \int_{\mu}^{+\infty} \frac{(s-\mu)^{n-2}}{a(\mu)}\left[f\left(s, x\left(g_{1}(s)\right), \ldots, x\left(g_{k}(s)\right)\right)-c(s)\right] d s d \mu, \quad t \geqslant \tau^{-1}(T)
\end{aligned}
$$

which leads that

$$
a(t)(x(t)+b(t) x(\tau(t)))^{\prime}=\frac{(-1)^{n}}{(n-2) !} \int_{t}^{+\infty}(s-t)^{n-2}\left[f\left(s, x\left(g_{1}(s)\right), \ldots, x\left(g_{k}(s)\right)\right)-c(s)\right] d s, \quad t \geqslant \tau^{-1}(T),
$$

which guarantees that

$$
\left[a(t)(x(t)+x(\tau(t)))^{\prime}\right]^{(n-1)}=(-1)\left[f\left(t, x\left(g_{1}(t)\right), \ldots, x\left(g_{k}(t)\right)\right)-c(t)\right], \quad t \geqslant \tau^{-1}(T),
$$

which together with (3.35) gives that $x$ is a bounded positive solution in $\overline{B(M, N)}$.

Next we show that Eq. (1.6) has uncountably many bounded positive solutions in $\overline{B(M, N)}$. Let $\mathrm{L}_{1}, \mathrm{~L}_{2} \in(M-N, M+N)$ and $\mathrm{L}_{1} \neq \mathrm{L}_{2}$. For every $\theta \in\{1,2\}$, we infer similarly that there exist constants $T_{L_{\theta}}, \beta_{\theta}$ and a mapping $S_{L_{\theta}}$ satisfying (3.35)-(3.37), where $L, \beta, T$ and $S_{L}$ are replaced by $L_{\theta}, \beta_{\theta}, T_{L_{\theta}}$ and $S_{L_{\theta}}$, respectively, and the mapping $S_{L_{\theta}}$ has a fixed point $x \in \overline{B(M, N)}$, which is a bounded positive solution of Eq. (1.6) in $\overline{\mathrm{B}(\mathrm{M}, \mathrm{N})}$, that is,

$$
\chi^{\theta}(t)=L_{\theta}+\frac{(-1)^{n+1}}{(n-2) !} \sum_{j=1}^{\infty} \int_{\tau^{-2 j+1}(t)}^{\tau^{-2 j}(t)} \int_{\mu}^{+\infty} \frac{(s-\mu)^{n-2}}{a(\mu)}\left[f\left(s, x^{\theta}\left(g_{1}(s)\right), \ldots, x^{\Theta}\left(g_{k}(s)\right)\right)-c(s)\right] d s d \mu, \quad t \geqslant T_{L_{\theta}} .
$$

In terms of (3.2), we get that there exists $T_{*}$ with $\tau^{-1}\left(T_{*}\right)>T_{*}>\max \left\{T_{L_{1}}, T_{L_{2}}\right\}$ satisfying

$$
\frac{1}{(\mathrm{n}-2) !} \int_{\mathrm{T}_{*}}^{+\infty} \int_{\mu}^{+\infty} \frac{\mathrm{s}^{\mathrm{n}-2}}{|\mathrm{a}(\mu)|} \mathrm{p}(\mathrm{s}) \mathrm{ds} \mathrm{d} \mu<\frac{\left|\mathrm{L}_{1}-\mathrm{L}_{2}\right|}{4} \text {. }
$$

In order to prove that the set of bounded positive solutions of Eq. (1.6) is uncountable, it is sufficient to verify that $x^{1} \neq x^{2}$. On account of (3.1), (3.43), and (3.44), we infer that for $t \geqslant T_{*}$

$$
\left|x^{1}(t)-x^{2}(t)\right|=\mid L_{1}-L_{2}+\frac{(-1)^{n+1}}{(n-2) !} \sum_{j=1}^{\infty} \int_{\tau^{-2 j+1}(t)}^{\tau^{-2 j}(t)} \int_{\mu}^{+\infty} \frac{(s-\mu)^{n-2}}{a(\mu)}
$$




$$
\begin{aligned}
& \times\left[f\left(s, x^{1}\left(g_{1}(s)\right), \ldots, x^{1}\left(g_{k}(s)\right)\right)-f\left(s, x^{2}\left(g_{1}(s)\right), \ldots, x^{2}\left(g_{k}(s)\right)\right)\right] d s d \mu \mid \\
\geqslant & \left|L_{1}-L_{2}\right|-\frac{1}{(n-2) !} \int_{\tau^{-1}(t)}^{+\infty} \int_{\mu}^{+\infty} \frac{(s-\mu)^{n-2}}{|a(\mu)|} \\
& \times\left[\left|f\left(s, x^{1}\left(g_{1}(s)\right), \ldots, x^{1}\left(g_{k}(s)\right)\right)\right|+\left|f\left(s, x^{2}\left(g_{1}(s)\right), \ldots, x^{2}\left(g_{k}(s)\right)\right)\right|\right] d s d \mu \\
\geqslant & \left|L_{1}-L_{2}\right|-\frac{2}{(n-2) !} \int_{\tau^{-1}\left(T_{*}\right)}^{+\infty} \int_{\mu}^{+\infty} \frac{s^{n-2}}{|a(\mu)|} p(s) d s d \mu \\
\geqslant & \left|L_{1}-L_{2}\right|-\frac{2}{(n-2) !} \int_{T_{*}}^{+\infty} \int_{\mu}^{+\infty} \frac{s^{n-2}}{|a(\mu)|} p(s) d s d \mu>\frac{\left|L_{1}-L_{2}\right|}{2},
\end{aligned}
$$

that is, $x^{1} \neq x^{2}$. This completes the proof.

Theorem 3.5. Let (C1), (C2), (C3), and (C4) hold. Assume that there exist two constants $\mathrm{M}$ and $\mathrm{N}$ with $\mathrm{M}>$ $\mathrm{N}>0$ and a function $\mathrm{p} \in \mathrm{C}\left(\left[\mathrm{t}_{0},+\infty\right), \mathbb{R}^{+}\right)$satisfying (3.1) and

$$
\begin{aligned}
\sum_{j=1}^{\infty} \int_{\tau^{-j}\left(t_{0}\right)}^{+\infty} \int_{\mu}^{+\infty} \frac{s^{n-2}}{|a(\mu)|}[p(s)+|c(s)|] d s d \mu & <+\infty, \\
b(t) & =-1, \text { eventually. }
\end{aligned}
$$

Then Eq. (1.6) has uncountably many bounded positive solutions.

Proof. Let $L \in(M-N, M+N)$. Now we prove that there exists a mapping $S_{L}: \overline{B(M, N)} \rightarrow C B([\beta,+\infty), \mathbb{R})$ with $S_{L}(\partial B(M, N)) \subset \overline{B(M, N)}$ such that $S_{L}$ has a fixed point $x \in \overline{B(M, N)}$, which is also a bounded positive solution of Eq. (1.6). It follows from (3.45), (3.46), (C3), and (C4) that there exist two constants $T$ and $\beta$ satisfying

$$
\begin{aligned}
& \tau^{-1}(T)>T>\beta \geqslant \max \left\{1,\left|t_{0}\right|+\left|\beta_{0}\right|,\left|\tau\left(t_{0}\right)\right|\right\}, \quad b(t)=-1, \quad t \geqslant \beta, \\
& \frac{2}{(n-2) !} \sum_{j=1}^{\infty} \int_{\tau^{-j}(\beta)}^{+\infty} \int_{\mu}^{+\infty} \frac{s^{n-2}}{|a(\mu)|}[p(s)+|c(s)|] d s d \mu<\min \{N+M-L, N-M+L\} .
\end{aligned}
$$

Define a mapping $S_{\mathrm{L}}: \overline{\mathrm{B}(\mathrm{M}, \mathrm{N})} \rightarrow \mathrm{CB}([\beta,+\infty), \mathbb{R})$ as follows:

$$
\left(S_{L} x\right)(t)= \begin{cases}L+\frac{(-1)^{n+1}}{(n-2) !} \sum_{j=1}^{\infty} \int_{\tau^{-j}(t)}^{+\infty} \int_{\mu}^{+\infty} \frac{(s-\mu)^{n-2}}{a(\mu)}\left[f\left(s, x\left(g_{1}(s)\right), \ldots, x\left(g_{k}(s)\right)\right)-c(s)\right] d s d \mu, & t \geqslant T, \\ \left(S_{L} x\right)(T), & \beta \leqslant t<T,\end{cases}
$$

for each $x \in \overline{\mathrm{B}(\mathrm{M}, \mathrm{N})}$. By (3.1), (3.13), (3.14), (3.48), and (3.49), we obtain that for any $x \in \partial B(M, N) \subset$ $\overline{\mathrm{B}(\mathrm{M}, \mathrm{N})}$ and $\mathrm{t} \geqslant \mathrm{T}$

$$
\begin{aligned}
\left|\left(S_{L} x\right)(t)-M\right| & =\left|L-M+\frac{(-1)^{n+1}}{(n-2) !} \sum_{j=1}^{\infty} \int_{\tau^{-j}(t)}^{+\infty} \int_{\mu}^{+\infty} \frac{(s-\mu)^{n-2}}{a(\mu)}\left[f\left(s, x\left(g_{1}(s)\right), \ldots, x\left(g_{k}(s)\right)\right)-c(s)\right] d s d \mu\right| \\
& \leqslant|L-M|+\frac{1}{(n-2) !} \sum_{j=1}^{\infty} \int_{\tau^{-j}(t)}^{+\infty} \int_{\mu}^{+\infty} \frac{(s-\mu)^{n-2}}{|a(\mu)|}\left[\left|f\left(s, x\left(g_{1}(s)\right), \ldots, x\left(g_{k}(s)\right)\right)\right|+|c(s)|\right] d s d \mu \\
& \leqslant|L-M|+\frac{1}{(n-2) !} \sum_{j=1}^{\infty} \int_{\tau^{-j}(T)}^{+\infty} \int_{\mu}^{+\infty} \frac{s^{n-2}}{|a(\mu)|}[p(s)+|c(s)|] d s d \mu \\
& \leqslant|L-M|+\frac{1}{(n-2) !} \sum_{j=1}^{\infty} \int_{\tau^{-j}(\beta)}^{+\infty} \int_{\mu}^{+\infty} \frac{s^{n-2}}{|a(\mu)|}[p(s)+|c(s)|] d s d \mu \\
& <|L-M|+\frac{1}{2} \min \{N+M-L, N-M+L\}<N,
\end{aligned}
$$

which means that $S_{\mathrm{L}}(\partial \overline{\mathrm{B}(\mathrm{M}, \mathrm{N})}) \subseteq \overline{\mathrm{B}(\mathrm{M}, \mathrm{N})}$. 
We claim that $S_{L}$ is a continuous, condensing mapping in $\overline{\mathrm{B}(M, N)}$. Put $\left\{x_{m}\right\}_{m \in \mathbb{N}} \subseteq \overline{\mathrm{B}(M, N)}$ and $x \in \overline{\mathrm{B}(M, N)}$ with $\lim _{m \rightarrow \infty} x_{m}=x$. Using (3.1), (3.48), (3.49), and the continuity of $f$, we infer that

$$
\begin{aligned}
& \left\|S_{L} x_{m}-S_{L} x\right\|=\sup _{t \geqslant T} \mid \frac{(-1)^{n+1}}{(n-2) !} \sum_{j=1}^{\infty} \int_{\tau^{-j}(t)}^{+\infty} \int_{\mu}^{+\infty} \frac{(s-\mu)^{n-2}}{a(\mu)}\left[f\left(s, x_{m}\left(g_{1}(s)\right), \ldots, x_{m}\left(g_{k}(s)\right)\right)\right. \\
& \left.-f\left(s, x\left(g_{1}(s)\right), \ldots, x\left(g_{k}(s)\right)\right)\right] d s d \mu \\
& \leqslant \frac{1}{(n-2) !} \sum_{j=1}^{\infty} \int_{\tau^{-j}(T)}^{+\infty} \int_{\mu}^{+\infty} \frac{s^{n-2}}{|a(\mu)|} \mid f\left(s, x_{m}\left(g_{1}(s)\right), \ldots, x_{m}\left(g_{k}(s)\right)\right) \\
& -f\left(s, x\left(g_{1}(s)\right), \ldots, x\left(g_{k}(s)\right)\right) \mid d s d \mu \\
& \leqslant \frac{1}{(n-2) !} \sum_{j=1}^{\infty} \int_{\tau^{-j}(\beta)}^{+\infty} \int_{\mu}^{+\infty} \frac{s^{n-2}}{|a(\mu)|} \mid f\left(s, x_{m}\left(g_{1}(s)\right), \ldots, x_{m}\left(g_{k}(s)\right)\right) \\
& -f\left(s, x\left(g_{1}(s)\right), \ldots, x\left(g_{k}(s)\right)\right) \mid d s d \mu, \quad m \in \mathbb{N}, \\
& \int_{\mu}^{+\infty} \frac{s^{n-2}}{|a(\mu)|}\left|f\left(s, x_{m}\left(g_{1}(s)\right), \ldots, x_{m}\left(g_{k}(s)\right)\right)-f\left(s, x\left(g_{1}(s)\right), \ldots, x\left(g_{k}(s)\right)\right)\right| d s \\
& \leqslant 2 \int_{\mu}^{+\infty} \frac{s^{n-2}}{|a(\mu)|} p(s) d s, \quad \mu \in\left[\tau^{-1}(\beta),+\infty\right)
\end{aligned}
$$

and

$$
\left|f\left(s, x_{m}\left(g_{1}(s)\right), \ldots, x_{m}\left(g_{k}(s)\right)\right)-f\left(s, x\left(g_{1}(s)\right), \ldots, x\left(g_{k}(s)\right)\right)\right| \rightarrow 0 \text { as } m \rightarrow \infty, \quad s \in\left[\tau^{-1}(\beta),+\infty\right),
$$

which together with the Lebesgue dominated convergence theorem gives that

$$
\lim _{m \rightarrow \infty} S_{L} x_{m}=S_{L} x
$$

that is, $S_{\mathrm{L}}$ is continuous in $\overline{\mathrm{B}(\mathrm{M}, \mathrm{N})}$.

By virtue of (3.1), (3.48), and (3.49), we deduce that for any $x \in \overline{\mathrm{B}(\mathrm{M}, \mathrm{N})}$

$$
\begin{aligned}
\left\|S_{L} x\right\|=\sup _{t \geqslant T}\left|S_{L} x(t)\right| & =\left|L+\frac{(-1)^{n+1}}{(n-2) !} \sum_{j=1}^{\infty} \int_{\tau^{-j}(t)}^{+\infty} \int_{\mu}^{+\infty} \frac{(s-\mu)^{n-2}}{a(\mu)}\left[f\left(s, x\left(g_{1}(s)\right), \ldots, x\left(g_{k}(s)\right)\right)-c(s)\right] d s d \mu\right| \\
& \leqslant L+\frac{1}{(n-2) !} \sum_{j=1}^{\infty} \int_{\tau^{-j}(T)}^{+\infty} \int_{\mu}^{+\infty} \frac{s^{n-2}}{|a(\mu)|}[p(s)+|c(s)|] d s d \mu \\
& \leqslant L+\frac{1}{(n-2) !} \sum_{j=1}^{\infty} \int_{\tau^{-j}(\beta)}^{+\infty} \int_{\mu}^{+\infty} \frac{s^{n-2}}{|a(\mu)|}[p(s)+|c(s)|] d s d \mu \\
& <L+\frac{1}{2} \min \{N+M-L, N-M+L\} \leqslant \frac{N+M+L}{2},
\end{aligned}
$$

which implies that $S_{L}(\overline{B(M, N)})$ is uniformly bounded in $[\beta,+\infty)$.

Let $\varepsilon>0$. Notice that (3.45) ensures that there exists $T^{*}>T$ satisfying

$$
\frac{1}{(n-2) !} \sum_{j=1}^{\infty} \int_{\tau^{-j}\left(T^{*}\right)}^{+\infty} \int_{\mu}^{+\infty} \frac{s^{n-2}}{|a(\mu)|}[p(s)+|c(s)|] d s d \mu<\frac{\varepsilon}{4} .
$$


It follows from (3.1), (3.49), and (3.50) that for all $x \in \overline{\mathrm{U}(M)}$ and $t_{2}>t_{1} \geqslant T^{*}$

$$
\begin{aligned}
\left|\left(S_{L} x\right)\left(t_{2}\right)-\left(S_{L} x\right)\left(t_{1}\right)\right| & \mid \frac{(-1)^{n+1}}{(n-2) !} \sum_{j=1}^{\infty} \int_{\tau^{-j}\left(t_{2}\right)}^{+\infty} \int_{\mu}^{+\infty} \frac{(s-\mu)^{n-2}}{a(\mu)}\left[f\left(s, x\left(g_{1}(s)\right), \ldots, x\left(g_{k}(s)\right)\right)-c(s)\right] d s d \mu \\
& -\frac{(-1)^{n+1}}{(n-2) !} \sum_{j=1}^{\infty} \int_{\tau^{-j}\left(t_{1}\right)}^{+\infty} \int_{\mu}^{+\infty} \frac{(s-\mu)^{n-2}}{a(\mu)}\left[f\left(s, x\left(g_{1}(s)\right), \ldots, x\left(g_{k}(s)\right)\right)-c(s)\right] d s d \mu \mid \\
\leqslant & \frac{1}{(n-2) !}\left\{\sum_{j=1}^{\infty} \int_{\tau^{-j}\left(t_{2}\right)}^{+\infty} \int_{\mu}^{+\infty} \frac{s^{n-2}}{|a(\mu)|}[p(s)+|c(s)|] d s d \mu\right. \\
& \left.+\sum_{j=1}^{\infty} \int_{\tau^{-j}\left(t_{1}\right)}^{+\infty} \int_{\mu}^{+\infty} \frac{s^{n-2}}{|a(\mu)|}[p(s)+|c(s)|] d s d \mu\right\} \\
\leqslant & \frac{2}{(n-2) !} \sum_{j=1}^{\infty} \int_{\tau^{-j}\left(T^{*}\right)}^{+\infty} \int_{\mu}^{+\infty} \frac{s^{n-2}}{|a(\mu)|}[p(s)+|c(s)|] d s d \mu<\varepsilon .
\end{aligned}
$$

On account of (3.2), (C2)-(C4), and Lemma 2.3, we get that there exists $H \in \mathbb{N}$ satisfying $\tau^{-H}(T)>T^{*}$. Set

$$
A_{3}=\min \left\{|a(t)|: t \in\left[\tau^{-j}(T), \tau^{-j}\left(T^{*}\right)\right], 1 \leqslant j \leqslant H\right\}
$$

and

$$
M_{4}=1+\frac{1}{(n-2) ! A_{3}} \int_{T}^{+\infty} s^{n-2}[p(s)+|c(s)|] d s .
$$

Since $\tau^{-j}$ is continuous on $\left[T, T^{*}\right]$ for $1 \leqslant j \leqslant H$, it follows that there exists $\delta>0$ satisfying

$$
\sup \left\{\left|\tau^{-j}\left(t_{1}\right)-\tau^{-j}\left(t_{2}\right)\right|: 1 \leqslant j \leqslant H, t_{1}, t_{2} \in\left[T, T^{*}\right],\left|t_{2}-t_{1}\right|<\delta\right\}<\frac{\varepsilon}{4 \mathrm{HM}_{4}},
$$

which together with (3.1), (3.49), (3.50), and (3.52) yields that for all $t_{1}, t_{2} \in\left[T, T^{*}\right], t_{1} \leqslant t_{2}<t_{1}+\delta$ and $x \in \overline{\mathrm{B}(M, N)}$

$$
\begin{aligned}
\left|\left(S_{L} x\right)\left(t_{2}\right)-\left(S_{L} x\right)\left(t_{1}\right)\right| & \mid \frac{(-1)^{n+1}}{(n-2) !} \sum_{j=1}^{\infty} \int_{\tau^{-j}\left(t_{2}\right)}^{+\infty} \int_{\mu}^{+\infty} \frac{(s-\mu)^{n-2}}{a(\mu)}\left[f\left(s, x\left(g_{1}(s)\right), \ldots, x\left(g_{k}(s)\right)\right)-c(s)\right] d s d \mu \\
& -\frac{(-1)^{n+1}}{(n-2) !} \sum_{j=1}^{\infty} \int_{\tau^{-j}\left(t_{1}\right)}^{+\infty} \int_{\mu}^{+\infty} \frac{(s-\mu)^{n-2}}{a(\mu)}\left[f\left(s, x\left(g_{1}(s)\right), \ldots, x\left(g_{k}(s)\right)\right)-c(s)\right] d s d \mu \mid \\
= & \frac{1}{(n-2) !}\left\{\mid \sum_{j=1}^{H} \int_{\tau^{-j}\left(t_{1}\right)}^{\tau^{-j}\left(t_{2}\right)} \int_{\mu}^{+\infty} \frac{(s-\mu)^{n-2}}{a(\mu)}\left[f\left(s, x\left(g_{1}(s)\right), \ldots, x\left(g_{k}(s)\right)\right)-c(s)\right] d s d \mu\right. \\
& +\sum_{j=H+1}^{\infty} \int_{\tau^{-j}\left(t_{1}\right)}^{+\infty} \int_{\mu}^{+\infty} \frac{(s-\mu)^{n-2}}{a(\mu)}\left[f\left(s, x\left(g_{1}(s)\right), \ldots, x\left(g_{k}(s)\right)\right)-c(s)\right] d s d \mu \\
& \left.-\sum_{j=H+1}^{\infty} \int_{\tau^{-j}\left(t_{2}\right)}^{+\infty} \int_{\mu}^{+\infty} \frac{(s-\mu)^{n-2}}{a(\mu)}\left[f\left(s, x\left(g_{1}(s)\right), \ldots, x\left(g_{k}(s)\right)\right)-c(s)\right] d s d \mu \mid\right\} \\
\leqslant & \frac{1}{(n-2) !}\left\{\sum_{j=1}^{H} \int_{\tau^{-j}\left(t_{1}\right)}^{\tau^{-j}\left(t_{2}\right)} \int_{\mu}^{+\infty} \frac{s^{n-2}}{|a(\mu)|}[p(s)+|c(s)|] d s d \mu\right. \\
& \left.+2 \sum_{j=1}^{\infty} \int_{\tau^{-j}\left(T^{*}\right)}^{+\infty} \int_{\mu}^{+\infty} \frac{s^{n-2}}{|a(\mu)|}[p(s)+|c(s)|] d s d \mu\right\} \\
\leqslant & H M_{4} \sup \left\{\left|\tau^{-j}\left(t_{1}\right)-\tau^{-j}\left(t_{2}\right)\right|: 1 \leqslant j \leqslant H, t_{1}, t_{2} \in\left[T, T^{*}\right],\left|t_{2}-t_{1}\right|<\delta\right\}+\frac{\varepsilon}{2}<\varepsilon .
\end{aligned}
$$


By means of (3.49), we infer that for all $x \in \overline{\mathrm{B}(\mathrm{M}, \mathrm{N})}$ and $t_{1}, t_{2} \in[\beta, T]$

$$
\left|\left(S_{L} x\right)\left(t_{2}\right)-\left(S_{L} x\right)\left(t_{1}\right)\right|=\left|\left(S_{L} x\right)(T)-\left(S_{L} x\right)(T)\right|<\varepsilon .
$$

It follows from (3.51), (3.53), and (3.54) that $S_{L}(\overline{B(M, N)})$ is equicontinuous in $[\beta,+\infty)$. Thus $S_{L}(\overline{B(M, N)})$ is relatively compact, that is, $S_{L}$ is condensing in $\overline{B(M, N)}$.

It follows from Lemma 2.2 that $S_{L}$ has a fixed point $x \in \overline{\mathrm{B}(M, N)}$, that is,

$$
x(t)=L+\frac{(-1)^{n+1}}{(n-2) !} \sum_{j=1}^{\infty} \int_{\tau^{-j}(t)}^{+\infty} \int_{\mu}^{+\infty} \frac{(s-\mu)^{n-2}}{a(\mu)}\left[f\left(s, x\left(g_{1}(s)\right), \ldots, x\left(g_{k}(s)\right)\right)-c(s)\right] d s d \mu, \quad t \geqslant \tau^{-1}(T),
$$

which gives that

$$
x(t)-x(\tau(t))=\frac{(-1)^{n}}{(n-2) !} \int_{t}^{+\infty} \int_{\mu}^{+\infty} \frac{(s-\mu)^{n-2}}{a(\mu)}\left[f\left(s, x\left(g_{1}(s)\right), \ldots, x\left(g_{k}(s)\right)\right)-c(s)\right] d s d \mu, \quad t \geqslant \tau^{-1}(T),
$$

which means that

$$
a(t)(x(t)-x(\tau(t)))^{\prime}=\frac{(-1)^{n+1}}{(n-2) !} \int_{t}^{+\infty}(s-t)^{n-2}\left[f\left(s, x\left(g_{1}(s)\right), \ldots, x\left(g_{k}(s)\right)\right)-c(s)\right] d s d \mu, \quad t \geqslant \tau^{-1}(T),
$$

and

$$
\left[a(t)(x(t)-x(\tau(t)))^{\prime}\right]^{(n-1)}=-f\left(t, x\left(g_{1}(t)\right), \ldots, x\left(g_{k}(t)\right)\right)+c(t), \quad t \geqslant \tau^{-1}(T),
$$

which together with (3.47) guarantees that $x \in \overline{\mathrm{B}(\mathrm{M}, \mathrm{N})}$ is a bounded positive solution of Eq. (1.6). The rest of the proof is similar to that of Theorems 3.4, and is omitted. This completes the proof.

Remark 3.6. Theorems 3.1-3.5 extend and improve Theorems 2.1-2.6 in [3], Theorem 1 in [5], and Theorems 2.1-2.5 in [4], respectively. The examples in Section 4 show that our results are indeed generalizations of the corresponding results in [3-5].

\section{Examples}

In this section we construct five examples to clarify the applications and superiority of the results presented in Section 3.

Example 4.1. Consider the following higher order nonlinear neutral differential equation

$$
\begin{aligned}
& {\left[t^{10}\left(x(t)+\frac{\left(t^{2}-2 t+\sqrt{1+t}\right) \sin t}{1+3 t^{2}} x(t-\sqrt{t})\right)^{\prime}\right]^{(n-1)}+\frac{t^{4} x^{3}\left(t^{2}\right) x^{2}(t-1)-t x^{2}(t+1)}{t^{n+6}+\left(t^{2}+1\right) \cos ^{2}\left(3 t^{3}\right)}} \\
& \quad=\frac{\sqrt{1+t^{2}}-t \sin \left(3 t^{2}\right)}{t^{n+3}}, \quad t \geqslant 1,
\end{aligned}
$$

where $\mathrm{n} \in \mathbb{N} \backslash\{1\}, \mathrm{t}_{0}=1$ and $\mathrm{k}=3$. Let $\mathrm{b}_{*}=\mathrm{b}^{*}=\frac{1}{3}, \mathrm{~N}=1, \mathrm{M}=4, \beta_{0}=0$ and

$$
\begin{array}{ll}
a(t)=t^{10}, & b(t)=\frac{\left(t^{2}-2 t+\sqrt{1+t}\right) \sin t}{1+3 t^{2}}, \quad \tau(t)=t-\sqrt{t}, \quad g_{1}(t)=t^{2}, \quad g_{2}(t)=t-1, \\
g_{3}(t)=t+1, & c(t)=\frac{\sqrt{1+t^{2}}-t \sin \left(3 t^{2}\right)}{t^{n+3}}, \quad f(t, u, v, w)=\frac{t^{4} u^{3} v^{2}-t w^{2}}{t^{n+6}+\left(t^{2}+1\right) \cos ^{2}\left(3 t^{3}\right)}, \\
p(t)=\frac{1024 t^{3}+16}{t^{n+5}}, & (t, u, v, w) \in\left[t_{0},+\infty\right) \times \mathbb{R}^{3} .
\end{array}
$$

It is easy to verify that (C1), (C2), and (3.1)-(3.3) are satisfied. Hence Theorem 3.1 implies that Eq. (4.1) has uncountably many bounded positive solutions. But the results in [3-5] are not applicable for Eq. (4.1). 
Example 4.2. Consider the following higher order nonlinear neutral differential equation

$$
\begin{aligned}
& \left.\left[t^{4} \ln \left(1+t^{2}\right)\left(x(t)+\frac{5+12 \ln ^{3} t}{1+\ln ^{3} t}\right) \times\left(t-\frac{1}{2 t}\right)\right)^{\prime}\right]^{(n-1)} \\
& \quad+\frac{t^{3} x^{2}\left(t^{2}-t\right) x(\sqrt{t})+\sqrt{\left|t x\left(\frac{t^{3}}{2}\right)\right|}}{t^{n+5}+\sin ^{2} t}=\frac{t^{2} \ln \left(1+\sqrt{t^{3}+1}\right)}{1+t^{n+4}}, \quad t \geqslant 1,
\end{aligned}
$$

where $n \in \mathbb{N} \backslash\{1\}, t_{0}=1$ and $k=3$. Let $b_{*}=5, b^{*}=12, N=1, M=13, \beta_{0}=0$ and

$$
\begin{array}{rlrl}
a(t) & =t^{4} \ln \left(1+t^{2}\right), & b(t)=\frac{5+12 \ln ^{3} t}{1+\ln ^{3} t}, & \tau(t)=t-\frac{1}{2 t^{\prime}}, g_{1}(t)= \\
g_{3}(t)=\frac{t^{3}}{2}, & c(t)=\frac{t^{2} \ln \left(1+\sqrt{t^{3}+1}\right)}{1+t^{n+4}}, & f(t, u, v, w)=\frac{t^{3} u^{2} v+\sqrt{|t w|}}{t^{n+5}+\sin ^{2} t}, \\
p(t)=\frac{2197 t^{3}+\sqrt{13 t}}{t^{n+5}}, & (t, u, v, w) \in\left[t_{0},+\infty\right) \times \mathbb{R}^{3} . &
\end{array}
$$

It is obvious that (C1)-(C3), (3.1), (3.2), and (3.18) are satisfied. Thus Theorem 3.2 ensures that Eq. (4.2) has uncountably many bounded positive solutions. But the results in [3-5] are not valid for Eq. (4.2).

Example 4.3. Consider the following higher order nonlinear neutral differential equation

$$
\begin{aligned}
& {\left[\left(t^{3}+1\right)(x(t)-(5-\cos t) x(\sqrt{t}))^{\prime}\right]^{(n-1)}+\frac{t^{4} x^{3}\left(t^{2} \ln t\right)+\left(t^{5}-3\right) x^{2}\left(t^{3}-1\right)}{t^{n-2}(1+t)^{8} \sqrt{1+x^{2}\left(t^{2}-t\right)}}} \\
& \quad=\frac{t^{3}-\sin \sqrt{4 t^{5}-t}}{t^{n+4}[3+\cos (t+\sqrt{t})]^{2}}, \quad t \geqslant 1,
\end{aligned}
$$

where $n \in \mathbb{N} \backslash\{1\}, t_{0}=1$ and $k=3$. Let $b_{*}=-6, b^{*}=-4, N=1, M=3, \beta_{0}=0$ and

$$
\begin{aligned}
& a(t)=t^{3}+1, \quad b(t)=-5+\cos t, \quad \tau(t)=\sqrt{t}, \quad g_{1}(t)=t^{2} \ln t, \quad g_{2}(t)=t^{3}-1, \\
& g_{3}(t)=t^{2}-t, \quad c(t)=\frac{t^{3}-\sin \sqrt{4 t^{5}-t}}{t^{n+4}[3+\cos (t+\sqrt{t})]}, \quad f(t, u, v, w)=\frac{t^{4} u^{3}+\left(t^{5}-3\right) v^{2}}{t^{n-2}(1+t)^{8} \sqrt{1+w^{2}}}, \\
& p(t)=\frac{9 t^{5}+27 t^{4}+27}{t^{n-2}(1+t)^{8}}, \quad(t, u, v, w) \in\left[t_{0},+\infty\right) \times \mathbb{R}^{3} .
\end{aligned}
$$

It is clear that (C1)-(C3), (3.1), (3.2), and (3.31) are satisfied. Therefore Theorem 3.3 means that Eq. (4.3) has uncountably many bounded positive solutions. But the results in [3-5] are inapplicable for Eq. (4.3).

Example 4.4. Consider the following higher order nonlinear neutral differential equation

$$
\begin{aligned}
& {\left[-t^{4}\left(x(t)+x\left(\frac{t}{2}\right)\right)^{\prime}\right]^{(n-1)}+\frac{x\left(t^{2}+1\right) x^{2}\left(t^{3} \ln (1+t)\right)}{t^{n+2}}-\frac{(t-1) x^{2}\left(t^{2}+1\right)}{t^{n+2}(1+t)(2+\sin t)}} \\
& =\frac{t^{2}+\left(1-3 t^{3}\right) \cos \left(t^{2}+t\right)}{t^{n+5}(t+1)(t+2)}, \quad t \geqslant 2,
\end{aligned}
$$

where $n \in \mathbb{N} \backslash\{1\}, t_{0}=2$ and $k=2$. Let $N=1, M=2, \beta_{0}=1$ and

$$
\begin{aligned}
& a(t)=-t^{4}, \quad b(t)=1, \quad \tau(t)=\frac{t}{2}, \quad g_{1}(t)=t^{2}+1, \quad g_{2}(t)=t^{3} \ln (1+t), \\
& c(t)=\frac{t^{2}+\left(1-3 t^{3}\right) \cos \left(t^{2}+t\right)}{t^{n+5}(t+1)(t+2)}, \quad f(t, u, v)=\frac{u v^{2}}{t^{n+2}}-\frac{(t-1) u^{2}}{t^{n+2}(1+t)(2+\sin t)}, \\
& p(t)=\frac{12}{t^{n+2}}, \quad(t, u, v) \in\left[t_{0},+\infty\right) \times \mathbb{R}^{2} .
\end{aligned}
$$

It is easy to see that (C1)-(C4), (3.1), (3.2), and (3.34) are satisfied. Therefore Theorem 3.4 guarantees that Eq. (4.4) has uncountably many bounded positive solutions. But the results in [3-5] are not valid for Eq. (4.4). 
Example 4.5. Consider the following higher order nonlinear neutral differential equation

$$
\begin{aligned}
& {\left[\left(t^{5}+1\right)^{\frac{3}{2}}(x(t)-x(\ln t))^{\prime}\right]^{(n-1)}+\frac{x^{2}\left(t^{2}+\ln t\right) x\left(t+\sqrt{1+t^{2}}\right)}{\left(1+t^{\frac{9}{2}}\right) t^{n-2}\left[1+\ln \left(1+x^{2}\left(t^{2}+\ln t\right)\right)\right]}} \\
& \quad=\frac{1+t^{3} \sin \left(t^{2}\right)}{t^{n+5}\left[\left(1+t^{4}\right)^{\frac{4}{3}}+\ln t\right]^{\prime}}, \quad t \geqslant 2,
\end{aligned}
$$

where $n \in \mathbb{N} \backslash\{1\}, t_{0}=2$ and $k=2$. Let $N=1, M=3, \beta_{0}=\ln 2$ and

$$
\begin{aligned}
& a(t)=\left(t^{5}+1\right)^{\frac{3}{2}}, \quad b(t)=-1, \quad \tau(t)=\ln t, \quad g_{1}(t)=t^{2}+\ln t, \quad g_{2}(t)=t+\sqrt{1+t^{2}}, \\
& c(t)=\frac{1+t^{3} \sin \left(t^{2}\right)}{t^{n+5}\left[\left(1+t^{4}\right)^{\frac{4}{3}}+\ln t\right]}, \quad f(t, u, v)=\frac{u^{2} v}{\left(1+t^{\frac{9}{2}}\right) t^{n-2}\left[1+\ln \left(1+u^{2}\right)\right]} \\
& p(t)=\frac{27}{\left(1+t^{\frac{9}{2}}\right) t^{n-2}}, \quad(t, u, v) \in\left[t_{0},+\infty\right) \times \mathbb{R}^{2} .
\end{aligned}
$$

It is easy to verify that (C1)-(C4), (3.1), (3.45), and (3.46) are satisfied. Thus Theorem 3.5 yields that Eq. (4.5) has uncountably many bounded positive solutions. But the results in [3-5] are inapplicable for Eq. (4.5).

\section{Acknowledgment}

This work was supported by the National Natural Science Foundation of China (Grant Number: 41701616).

\section{References}

[1] R. P. Agarwal, S. R. Grace, D. O'Regan, Nonoscillatory solutions for discrete equations, Comput. Math. Appl., 45 (2003), 1297-1302. 1, 1, 2.1

[2] K. Deimling, Nonlinear Functional Analysis, Springer-Verlag, Berlin (1985). 2.2

[3] Z. Liu, H. Gao, S. M. Kang, S. H. Shim, Existence and mann iterative approximations of nonoscillatory solutions of nth order neutral delay differential equations, J. Math. Anal. Appl., 329 (2007), 515-529. 1, 1, 3.6, 4.1, 4.2, 4.3, 4.4, 4.5

[4] Z. G. Zhang, A. J. Yang, C. N. Di, Existence of positive solutions of second order nonlinear neutral differential equations with positive and negative terms, J. Appl. Math. Comput., 25 (2007), 245-253. 1, 3.6

[5] Y. Zhou, Existence for nonoscillatory solutions of second order nonlinear differential equations, J. Math. Anal. Appl., 331 (2007), 91-96. 1, 1, 3.6, 4.1, 4.2, 4.3, 4.4, 4.5

[6] X. L. Zhou, R. Yu, Oscillatory behavior of higher order nonlinear neutral forced differential equations with oscillating coefficients, Comput. Math. Anal., 56 (2008), 1562-1568. 1

[7] Y. Zhou, B. G. Zhang, Y. Q. Huang, Existence for nonoscillatory solutions of higher order nonlinear neutral differential equations, Czechoslovak Math. J., 55 (2005), 237-253. 1, 1, 1 